\title{
Investigación, desarrollo e innovación en el sector empresarial español: dificultades para su implementación
}

Research, development and innovation in the Spanish business sector: difficulties for its implementation

\author{
María R. Osuna-Alarcón; Pilar Rodríguez-Hernández
}

Cómo citar este artículo:

Osuna-Alarcón, María R.; Rodríguez-Hernández, Pilar (2020). “Investigación, desarrollo e innovación en el sector empresarial español: dificultades para su implementación”. El profesional de la información, v. 29, n. 1, e290119.

https://doi.org/10.3145/epi.2020.ene.19

Artículo recibido el 09-06-2019

Aceptación definitiva: 18-11-2019

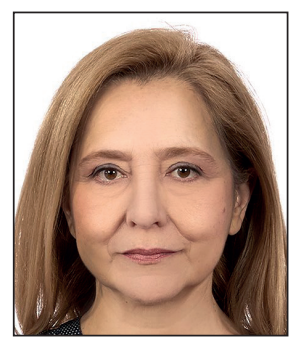

María R. Osuna-Alarcón

https://orcid.org/0000-0002-0099-1065

Universidad de Salamanca

Facultad de Traducción y Documentación

Dpto. de Biblioteconomía y Documentación

Francisco de Vitoria, 6-16.

37008 Salamanca, España

osuna@usal.es

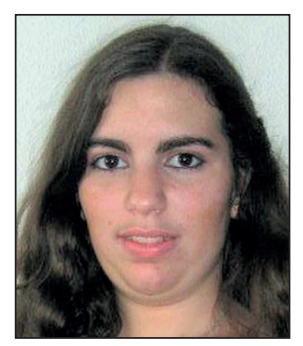

Pilar Rodríguez-Hernández

https://orcid.org/0000-0003-2760-8051

Universidad de Salamanca

Facultad de Traducción y Documentación

Instituto de Estudios de la Ciencia y la

Tecnología (ECYT-USAL).

Francisco de Vitoria, 6-16.

37008 Salamanca, España

pilar_92@usal.es

\section{Resumen}

Para conocer las dificultades percibidas y reales de la empresa en España en la implementación de la l+D+i por el tejido empresarial, vamos a partir de la visión que tienen los empleados sobre la facilidad o dificultad de sus empresas a la hora de hacer investigación y desarrollo y, por tanto, innovación. En este trabajo analizamos los aspectos más destacados de la encuesta elaborada bajo los auspicios del "Proyecto de investigación cultura científica: percepción y actitudes ante la ciencia y la innovación en el sector empresarial español" financiado por el Ministerio de Economía y Competitividad de España. Los resultados obtenidos adelantan que la educación y la promoción de una actitud innovadora son la base para conseguir un desarrollo exitoso empresarial ya que hoy la innovación resulta uno de los pilares clave del mantenimiento y supervivencia de las empresas.

\section{Palabras clave}

Investigación; Desarrollo; Innovación; Educación en innovación; I+D+i; Industrias; Sectores industriales; Percepción de la ciencia; Actitudes; Barreras; Cultura científica; Empresas españolas; Estadísticas; España.

\begin{abstract}
To know the perceived and real difficulties of the company in Spain in relation to the implementation of $R+D+i$ by the Spanish business, we start from the vision that employees have about the ease or difficulty of their companies at the time of doing research and development and therefore, innovation. In this paper we analyze the highlights of the survey prepared under the auspices of the "Scientific Culture Research Project: perception and attitudes towards science and innovation in the Spanish business sector" funded by the Ministry of Economy and Competitiveness. The results obtained advance that education and the promotion of an innovative attitude are the basis for achieving a successful business development since innovation is one of the key pillars of the maintenance and survival of companies today.
\end{abstract}

\section{Keywords}

Research; Investigation; Development; Innovation; Innovation education; RDi; R\&D; Industries; Industrial sectors; Perception of science; Attitudes; Barriers; Scientific culture; Spanish companies; Statistics; Spain. 


\section{Introducción}

El éxito y en muchos casos la supervivencia de una empresa depende del esfuerzo que ésta dedica a la innovación, la competitividad, la eficacia y a la gestión. Mejorar la estrategia comparativa en estos ámbitos se hace imprescindible.

Según la Cámara de Comercio de España, para ser efectiva la competitividad debe sustentarse en la innovación:

"La innovación es el medio a través del cual se crean nuevos recursos generadores de riqueza o dotan a los recursos existentes de mayor potencial para crearla, es una clara respuesta a los desafíos económicos actuales, particularmente a la hora de competir en un mundo globalizado, sin fronteras. Sin embargo, uno de los problemas más acuciantes de nuestro país es el reducido número de empresas y emprendedores que basan su competitividad en innovación" (Cámara de Comercio de España, 2017).

Esta afirmación de la Cámara de Comercio de España sobre sus propios miembros resulta un hecho constatable cuando se analizan las encuestas nacionales e internacionales sobre la innovación en el sector empresarial español.

“Cuando se analiza en las series estadísticas de innovación, generadas por el INE, el peso de las empresas innovadoras en el conjunto, llama la atención cómo en los últimos años desciende paulatinamente el número de las pymes que se consideran innovadoras, en contraste con el alza que registra dicha consideración entre las grandes empresas. Esta información nos pone ante la evidencia no sólo de que la presencia de la innovación es algo aún lejano para la mayoría de las empresas, sino también de que diversos factores están retrayendo la penetración de la innovación precisamente en el tejido más potente en la economía española, las pymes" (González-Hermoso-de-Mendoza; Sánchez-López, 2010, p. 255).

Consecuentemente, podemos observar cómo el valor de la innovación en el sector empresarial no es algo cercano ni inmediato y es que según el Manual de Frascati la I+D comprende

"el trabajo creativo llevado a cabo de forma sistemática para incrementar el volumen de conocimientos, incluido el conocimiento del hombre, la cultura y la sociedad, y el uso de esos conocimientos para crear nuevas aplicaciones" (OCDE, 2003).

Sobre la base de estas definiciones de I+D+i trataremos de examinar las variables culturales que influyen en las actividades del sector empresarial en materia de I+D y en su comportamiento innovador, para posteriormente analizar las dificultades y obstáculos percibidos para implementarla en las empresas a través de los datos obtenidos por el proyecto de investigación Cultura científica empresarial.

La motivación de este trabajo se centra en la constatación de que el sector empresarial ha cobrado un creciente papel en la escena de los planes y políticas públicas de ciencia y tecnología, tanto nacionales como europeas.

"Este hecho contrasta en la práctica con un escenario en el que se ha detectado la denominada brecha entre investigación y mercado. Este fenómeno está definido por una escasa participación empresarial en la financiación y ejecución de la I+D+i, especialmente en la modalidad de cooperación con el sector público, y falta de estrategias para la apropiación por parte de las empresas del conocimiento generado por universidades y centros públicos de investigación" (López-Navarro et al., 2016).

Como hipótesis de partida se ha podido constatar que

"numerosas fuentes avalan con datos cuantitativos este escenario. Se trata fundamentalmente de medidas resultantes de enfoques economicistas, orientados a medir la cantidad de dinero que emplean las empresas con fines de I+D+i, el número de contactos con universidades o las patentes registradas, por ejemplo" (Grupo de Investigación en Evaluación y Transferencia Científica, 2017).

En el proyecto se planteó la necesidad de complementar estos datos con un análisis de la percepción del sector empresarial que aportase conocimiento sobre los aspectos sociológicos que no se han cubierto hasta la fecha:

"el presente proyecto ha partido de la hipótesis de que la percepción de la ciencia y la innovación, así como la cultura científica empresarial, están relacionadas con este tipo de comportamientos que tienen que ver con las actividades innovadoras, tiene como objetivo examinar las variables subjetivas y culturales que influyen en las actividades del sector empresarial en materia de I+D+i y en su comportamiento innovador. Se pretende estudiar cuál es la percepción de la ciencia, la tecnología y la innovación en el sector empresarial y cuáles son las actitudes y motivaciones de las empresas españolas hacia la financiación y ejecución de la I+D+i (tanto interna como en cooperación con el sector público) así como los principales obstáculos para la apropiación de conocimiento científico" (Grupo de Investigación en Evaluación y Transferencia Científica, 2017).

En los últimos años el campo de los estudios de la percepción social de la ciencia y la tecnología ha experimentado importantes cambios, siendo destacable señalar la Encuesta de percepción social de la ciencia de la Fundación Española Para la Ciencia y la Tecnología (Fecyt, 2018) y los Eurobarómetros de ciencia y tecnología de la Comisión Europea (Comisión Europea, 2005a; 2005b; 2007; 2010b).

La divulgación científica tiene poca trayectoria histórica en los programas nacionales de investigación y desarrollo. Sin 
embargo, el gobierno español está poniendo de su parte para revertir esta situación (Fecyt, 2007), como demuestra la definición de:

- una Acción Estratégica para la divulgación de la ciencia y la tecnología dentro del área de investigación básica no orientada en el Plan Nacional de I+D+i 2000-2003, cuya continuidad se establece en el Plan Estatal de Investigación Científica y Técnica y de Innovación 2017-2020, actualmente en vigor;

- Estrategia española de ciencia y tecnología y de innovación 2013-2020 sobre el fomento de la cultura científica y tecnológica de la sociedad (Ministerio de Ciencia, Innovación y Universidades, 2019b);

- políticas en relación con el Programa marco de investigación e innovación de la Unión Europea, Horizonte 2020 (H2020) que cubre el período 2014-2020, financia proyectos de investigación e innovación de diversas áreas temáticas en el contexto europeo (Ministerio de Ciencia, Innovación y Universidades, 2019a), y que cuenta con un presupuesto de 74.800 millones de euros (Comisión Europea, 2010c).

Adentrándonos en el ámbito empresarial objeto de estudio partimos de la Encuesta sobre innovación en las empresas realizada por el Instituto Nacional de Estadística (INE) que tiene como principal objetivo ofrecer información directa sobre el proceso de innovación en las empresas (INE, 2019). A este respecto, y teniendo en cuenta que nuestro objetivo es conocer la innovación en las empresas españolas, es fundamental mencionar también el Índice mundial de innovación de 2019, publicado por la Organización Mundial de la Propiedad Intelectual, que clasifica y desglosa el desempeño en innovación de las economías mundiales anualmente (Dutta; Lanvin; Wunsch-Vincent, 2019); así como el Índice de competitividad global de 2018 elaborado y publicado anualmente desde 1979 por el Foro Económico Mundial (World Economic Forum, 2019).

"La I+D (investigación científica y desarrollo tecnológico) y la innovación son valores estratégicos con importantes y muy diversas repercusiones para las sociedades actuales. Los datos disponibles insisten en demostrar que las economías más dinámicas y exitosas, las que han conseguido acelerar sus tasas de crecimiento y abrir una brecha tecnológica respecto a los países menos activos, pero también las que están consiguiendo aumentar los provechos para sus ciudadanos, no son precisamente las que compiten en base a la reducción de costes y salarios, a las políticas de austeridad y recortes, sino invirtiendo en formación y en conocimiento, en investigación y en innovación. No es que los países más ricos inviertan más en ciencia y en investigación, sino que los que invirtieron en ciencia e investigación son ahora los más ricos, en términos económicos y sociales" (Rey-Rocha et al., 2018).

\section{Metodología}

El presente trabajo parte de la encuesta Cultura científica empresarial y se adentra en la percepción de la ciencia y la innovación por la empresa española en general. Tiene como objetivo examinar las dificultades para implementar la innovación, los obstáculos para invertir e implementar la I+D, así como los factores que afectan a la innovación en España desde la perspectiva de las empresas españolas. Es decir, se han examinado las variables que influyen en las actividades del sector empresarial en I+D+i y en su comportamiento innovador para determinar cuáles son las motivaciones de las empresas españolas hacia la ejecución de las políticas de I+D+i. Destacaremos además los aspectos más relevantes de las fuentes de información, para estudiar el conocimiento científico desde la cultura científica empresarial como parte de la información y documentación. La encuesta en cuestión es: Cultura científica empresarial en España 2016. Cultura científica, percepción y actitudes ante la ciencia y la innovación en el sector empresarial español; encuesta CCe (Rey-Rocha; López-Navarro, 2016; Cultura Científica Empresarial, 2017; Rey-Rocha et al., 2018) del proyecto de investigación Cultura científica empresarial; Proyecto CCe (Proyecto CSO2014-53293-R) ${ }^{1}$. Se articula en torno a la realización de una encuesta telefónica con cuestionario estructurado de percepción de la ciencia dirigida a empresas nacionales seleccionadas a partir de la base de datos SABI (Sistema de análisis de balances ibéricos) a fecha diciembre de 2014, que se llevó a cabo del 20 de septiembre al 11 de octubre de $2016^{2}$.

Según los datos de la propia encuesta CCe (Rey-Rocha et al., 2018) el tamaño muestral es de 707 casos, seleccionados mediante un diseño por conglomerados y estratificado por: tamaño (microempresas, pymes y grandes empresas) y sector de actividad ${ }^{3}$, cuyo error muestral es de $\pm 3,7 \%$. La encuesta se centra en el sector empresarial, pero incluyendo profesionales con distinto grado de relación, responsabilidad y capacidad de decisión en relación con la l+D+i.

Tabla 1. Universo objeto de la encuesta (datos encuesta CCe)

\begin{tabular}{|c|c|c|c|c|c|c|}
\hline & & \multicolumn{5}{|c|}{ Tamaño de la empresa (número de empleados) } \\
\hline & & $\begin{array}{l}\text { Micro empresa } \\
\qquad 10\end{array}$ & $\begin{array}{c}\text { Pequeña empresa } \\
\qquad 10-49\end{array}$ & $\begin{array}{c}\text { Mediana empresa } \\
50-249\end{array}$ & $\begin{array}{l}\text { Gran empresa } \\
250 \text { o más }\end{array}$ & TOTAL \\
\hline \multirow{5}{*}{ Sector } & Agricultura & 11.985 & 2.962 & 331 & 48 & 15.326 \\
\hline & Industria & 39.330 & 16.010 & 3.074 & 579 & 58.993 \\
\hline & Energía & 2.331 & 652 & 185 & 93 & 3.261 \\
\hline & Construcción & 51.998 & 7.738 & 690 & 120 & 60.546 \\
\hline & Servicios & 255.485 & 48.465 & 7.445 & 1.660 & 313.055 \\
\hline Total & & 361.129 & 75.827 & 11.725 & 2.500 & 451.181 \\
\hline
\end{tabular}


El cuestionario, específicamente diseñado para su administración vía telefónica, se compone de:

a) Veinte preguntas que recogen información sobre las cuatro dimensiones identificadas por el Manual de Antigua: Indicadores de percepción pública de la ciencia y la tecnología (Ricyt, 2015):

- institucional de la ciencia y la tecnología;

- hábitos informativos y culturales sobre ciencia y tecnología;

- actitudes y valores en relación con ciencia y tecnología;

- apropiación de la ciencia y la tecnología.

b) Datos sociodemográficos de la persona informante: sexo, edad, nivel de estudios y cargo en la empresa.

c) Tres preguntas sobre las características estructurales de la empresa y su relación con el entorno.

Vamos a analizar a través de los datos de dicha encuesta, el significado que tiene la innovación en el espacio físico y temporal demarcado por la misma. Además, nos apoyamos en otros estudios sobre la percepción de la ciencia como son los de Wynne (1991); Einsiedel (2000); Eizaguirre (2009) y López-Navarro et al. (2016).

\section{Dificultades para implementar la innovación en España desde la encuesta CCe}

Para conocer las dificultades percibidas y encontradas en relación con la implementación de la l+D+i por el tejido empresarial español, debemos partir de la visión que tienen los empleados sobre la facilidad o dificultad de sus empresas para hacer investigación y desarrollo y por tanto innovación. En este trabajo aceptamos la muestra seleccionada por la encuesta elaborada bajo los auspicios del proyecto de investigación Cultura científica: percepción y actitudes ante la ciencia y la innovación en el sector empresarial español"; en adelante Proyecto CCe (2017); en la que se realizaron 707 entrevistas a empleados de empresas españolas, considerándola lo suficientemente amplia y representativa como para que los valores obtenidos nos permitan hacer una extrapolación al resto de la cultura científica de las empresas españolas.

Así, en la encuesta elaborada por el Proyecto CCe se pregunta: ¿Cree usted que, para las empresas, hacer investigación y desarrollo o I+D en España es muy fácil, bastante fácil, bastante difícil o muy difícil? A esta pregunta; y tomando los datos totales; observamos cómo el $47,8 \%$ de los encuestados cree que es bastante difícil hacer investigación y desarrollo en España. Pero, es más, el 71,4\% de los encuestados respondió que es bastante o muy difícil para las empresas hacer I+D en España frente al 9,3\% de los que sí creen que es bastante fácil (gráfico 1).

La primera dificultad a la que se enfrentan las empresas en España es la creencia de sus directivos de que en su empresa es difícil hacer investigación y desarrollo y por tanto innovación. Y si los directivos no desarrollan de forma inicial una política de I+D+i porque lo consideran en general y en su empresa en particular bastante o muy difícil, resultaría sorprendente que esta empresa contara con un plan estratégico de I+D+i. Actualmente y debido a que vivimos en un entorno empresarial muy competitivo, los organigramas de las empresas están cambiando, no sólo en relación con las funciones y responsabilidades que cada dirección o equipo directivo asume, sino también en la forma en la que nos referimos a esos cargos a través, por ejemplo, de tecnicismos y neologismos ${ }^{4}$.

La encuesta CCe nos muestra esta percepción de complejidad a la hora de implementar la I+D+i entre los puestos organizativos. Para el $44,9 \%$ de los directores generales, el $66,7 \%$ de los directores técnicos y el $47,8 \%$ de los directores de producción, fábrica e instalaciones es bastante difícil hacer I+D. También es considerado bastante difícil con un $72,3 \%$ para los propios responsables de investigación.

Entre los resultados por sectores de actividad empresarial la conclusión común es que para todos los sectores es bastante o muy difícil hacer I+D en España (gráfico 2). Estos resultados los debemos poner en relación con el nivel de estudios de los encuestados. Se comprueba que a mayor nivel de es-

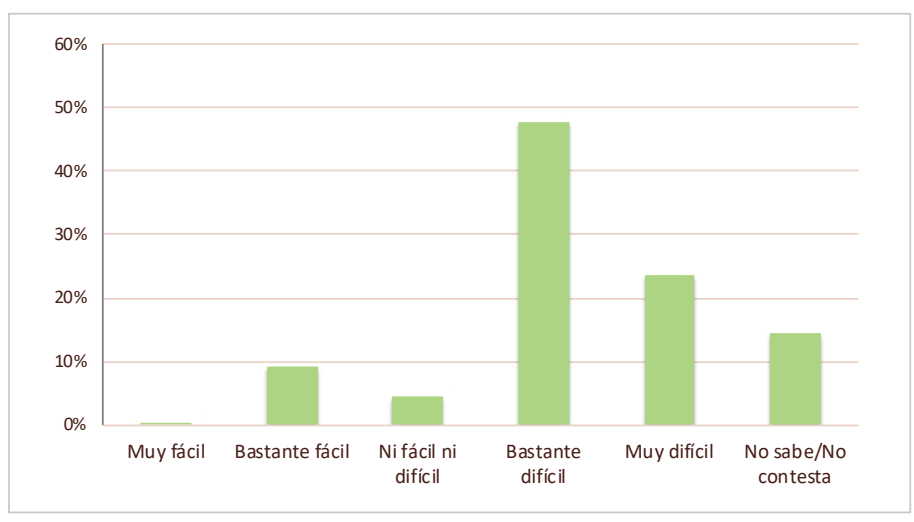

Gráfico 1. ¿Cree usted que, para las empresas, hacer investigación y desarrollo o I+D en España es muy fácil, bastante fácil, bastante difícil o muy difícil? (datos encuesta $C(e)$. 
tudios se percibe menor complejidad para la implantación de políticas de I+D en la empresa. De hecho, es llamativo cómo el nivel de estudios es un factor que afecta de forma determinante la I+D+i empresarial ya que como se observa en el gráfico 3, más del 50\% de los encuestados con un nivel de estudios universitario creen que es bastante difícil hacer I+D en su empresas, mientras que los empleados con un nivel de estudios primarios y medios han preferido optar mayoritariamente por la opción "no sabe" acerca de la dificultad de implementar la I+D+i en las empresas a la hora de responder a la misma pregunta (gráfico 3).

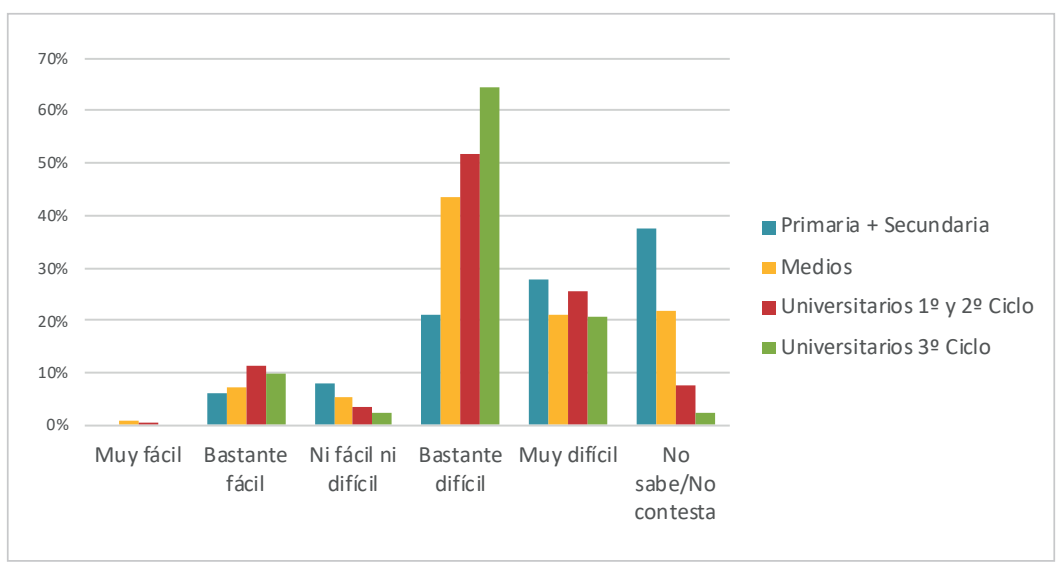

Gráfico 3. ¿Cree usted que, para las empresas, hacer investigación y desarrollo o I+D en España es muy fácil, bastante fácil, bastante difícil o muy difícil? Estudios (datos encuesta CCe).

Una vez identificadas las creencias de los encuestados sobre la implementación de la I+D+i, y teniendo en cuenta que la mayoría considera que en España es bastante o muy difícil hacer I+D+i, pasaremos a ver más de cerca las dificultades percibidas por estos mismos entrevistados sobre el porqué de su no implementación través de la siguiente pregunta de la encuesta: ¿Cuáles son los motivos principales por los que su empresa nunca ha intentado abordar actividades de investigación, desarrollo e innovación, o I+D+i?

Lo primero que observamos es que la falta de conocimiento y de los recursos técnicos suficientes $(3,0 \%)$ es una dificultad real en las empresas que no han intentado nunca abordar actividades de I+D+i (gráfico 4).

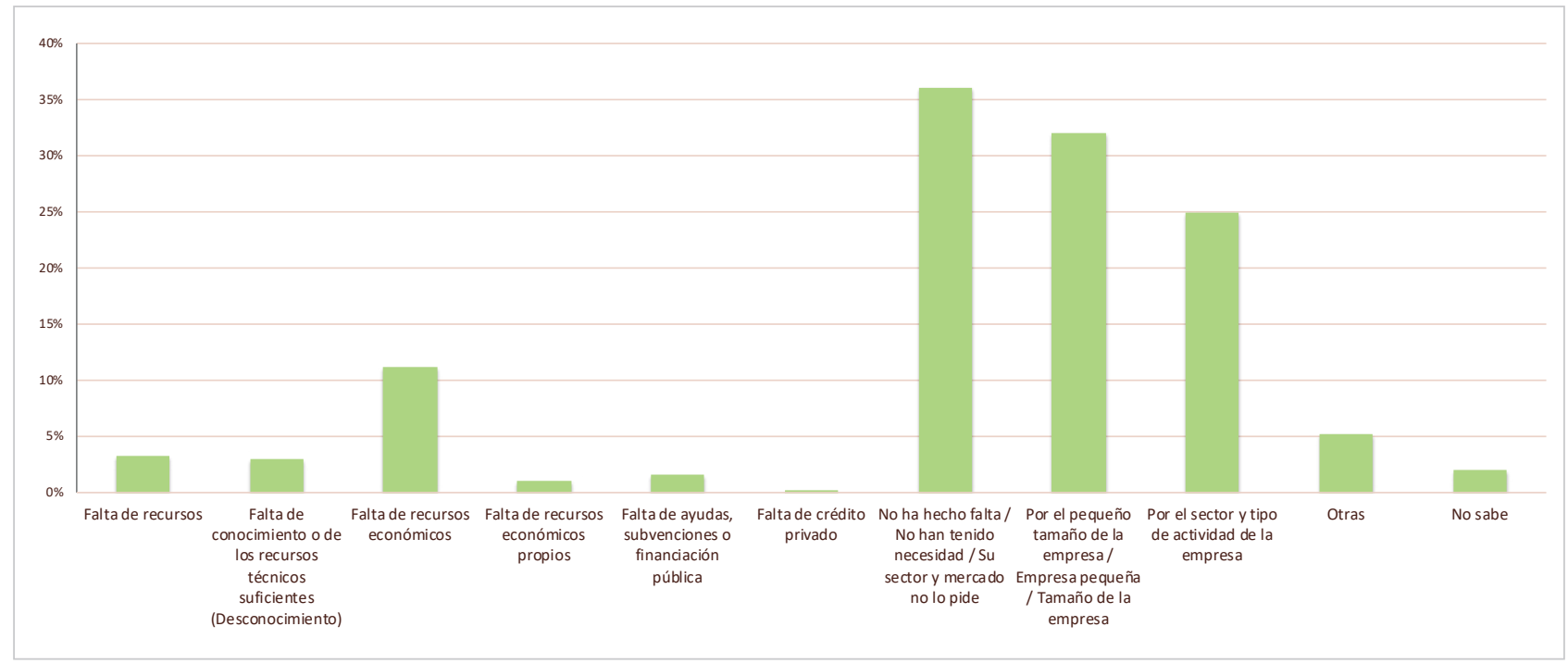

Gráfico 4. ¿Cuáles son los motivos principales por los que su empresa nunca ha intentado abordar actividades de investigación, desarrollo e innovación, o I+D+i? (datos encuesta CCe).

Llama la atención que la falta de recursos económicos -ya sean propios, públicos o privados- no es el principal escollo para la no implementación de la I+D+i en su empresa. También son factores determinantes que afectan a la I+D y más concretamente a la no innovación, los que están relacionados directamente con el sector de la empresa y el tipo de actividad que desempeñan (36,0\% y $24,8 \%$ respectivamente) así como con el tamaño de la empresa y el número de empleados a su cargo (31,9\%). Por lo que podemos apuntar así mismo que el tamaño de la empresa y el sector en el que trabajan y llevan a cabo sus actividades son factores determinantes para la no implementación de la I+D+i, y no son tan determinantes la falta de medios o recursos económicos y financieros. Las grandes empresas de sectores más científicos y tecnológicos son las que implementan I+D+i entre sus objetivos (tabla 2).

En este punto, y una vez identificadas las dificultades por las cuales no se ha implementado la I+D+i, vamos a analizar cuál es su grado de implicación en la innovación: ¿Hasta qué punto se considera usted interesado/a en los avances en la ciencia y tecnología aplicados a su sector? (gráfico 5).

Esta pregunta es fundamental porque a partir del interés que muestren los encuestados hacia los avances en la ciencia y la tecnología aplicados a su sector, podemos acercarnos a la su percepción de cómo la ciencia y la tecnología pue- 
Tabla 2. ¿Cuáles son los motivos principales por los que su empresa nunca ha intentado abordar actividades de investigación, desarrollo e innovación, o I+D+i? Sector y tamaño empresa (datos encuesta $C C e$ ).

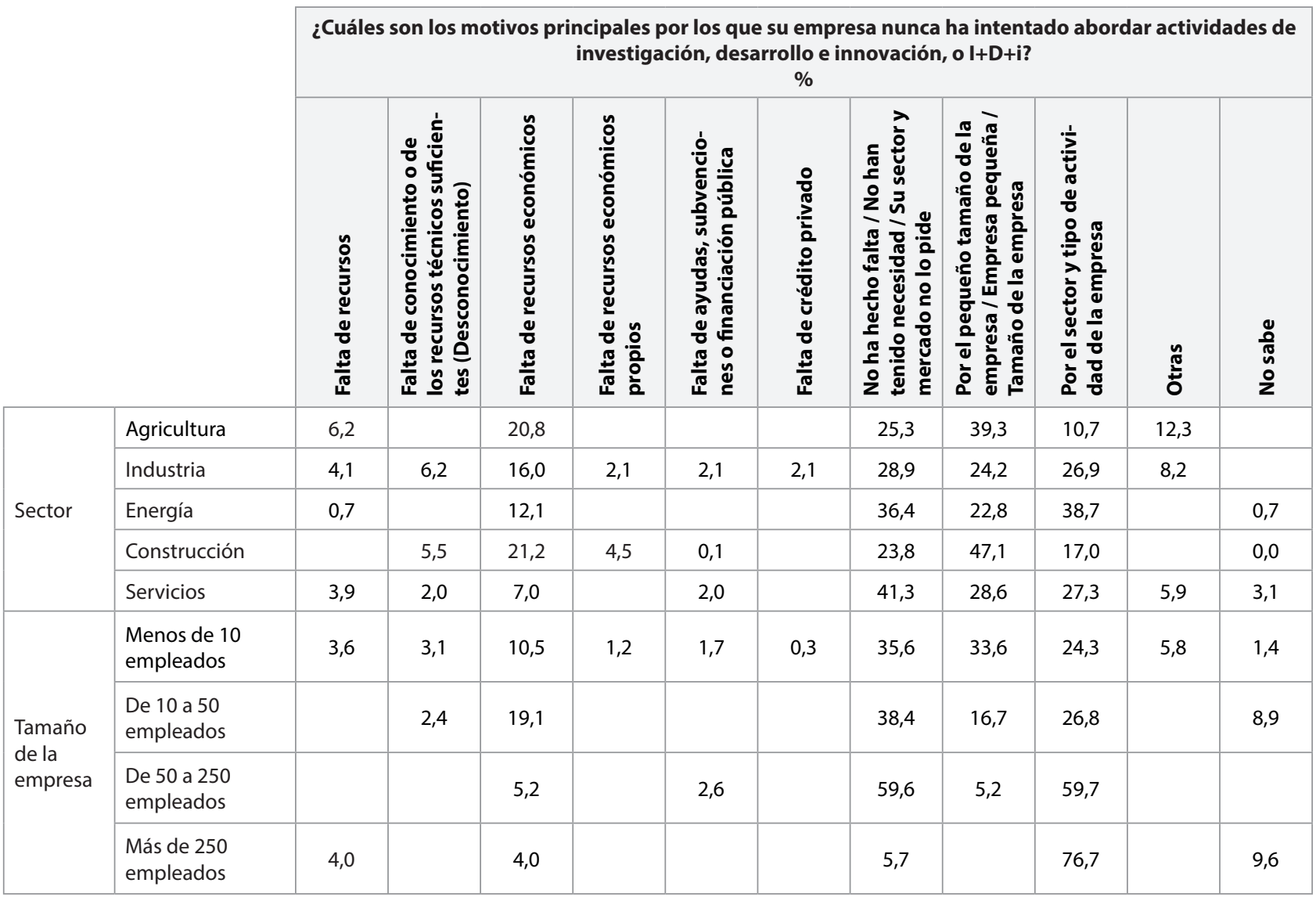

de favorecer el desarrollo empresarial. Podemos ver que el $70,7 \%$ de los encuestados sí que está bastante o muy interesado en los avances en la ciencia y tecnología de su sector empresarial.

Si vemos estos porcentajes por sectores empresariales las diferencias son considerables. Como ya hemos señalado, uno de los factores que afectan a la innovación es el sector empresarial en el que se encuadra la empresa, así como el tamaño de la misma (gráfico 4).

Por sectores podemos comprobar que el sector de la construcción es el que menos está interesado en los avances científico-tecnológicos que afectan la implementación de la I+D+i. Por el contrario, el sector energético es el más interesado en estos avances y por tanto el que más posibilidades tiene de implementar una política de I+D+i (gráfico 6). Como hemos visto hasta aquí, el sector, el tamaño de la empresa y la formación de sus directivos inciden directamente en el desarrollo de políticas de innovación empresariales además otra serie de esfuerzos. El estar interesado en los avances de la ciencia y a tecnología es el punto de partida para ello, ya que la

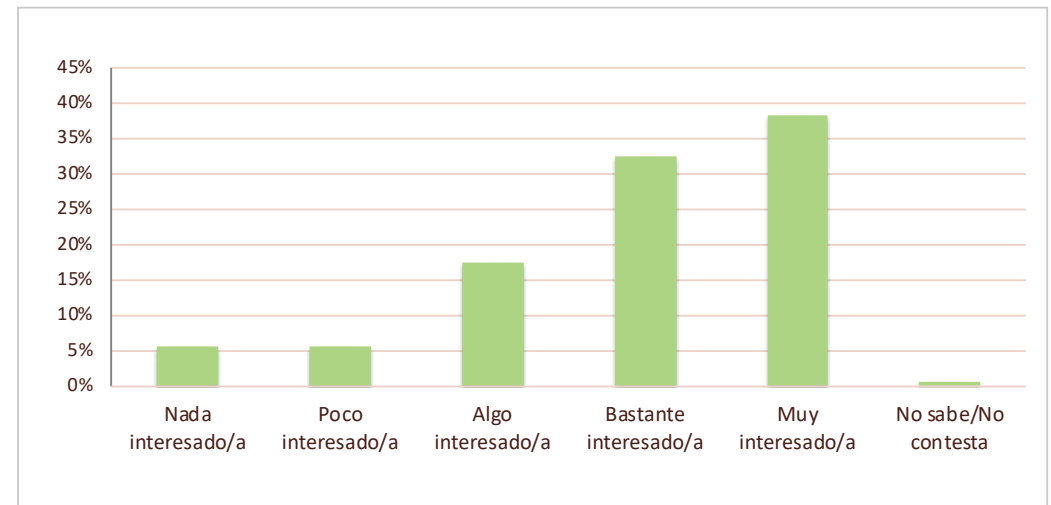

Gráfico 5. ¿Hasta qué punto se considera usted interesado/a en los avances en la ciencia y tecnología aplicados a su sector? (datos encuesta $\mathrm{CC}$ ).

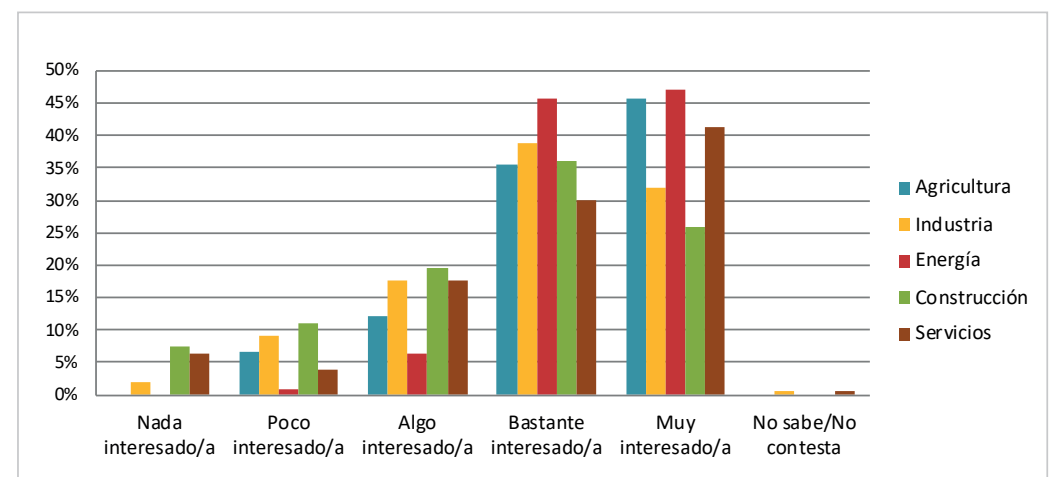

Gráfico 6. ¿Hasta qué punto se considera usted interesado/a en los avances en la ciencia y tecnología aplicados a su sector? Sector (datos encuesta CCe). 
innovación consiste en ser capaces de convertir los resultados de las investigaciones científico-técnicas en servicios y productos nuevos y mejores, a fin de seguir siendo competitivos en el mercado mundial (Gouardères, 2019).

Este hecho se constata también en las empresas con un número de empleados mayor, siendo las que más interesadas están en los avances de la ciencia y tecnología aplicados a su sector empresarial. Comprobamos cómo el $43,2 \%$ de las empresas de más de 250 empleados están muy interesadas en estos avances científico tecnológicos cifra que se eleva hasta el $82,0 \%$ si tenemos en cuenta que estas mismas empresas están bastante o muy interesadas en estos avances de su sector. Lo mismo sucede con las empresas que cuentan en su plantilla con 50 a 250 empleados, ya que el $82,4 \%$ de los trabajadores de este tamaño de empresa han contestado que están bastantes o muy interesados en los avances de la ciencia y la tecnología aplicados a su sector. Al contrario, podemos observar que, a menor número de empleados, menor es el interés por estos avances (gráfico 7).

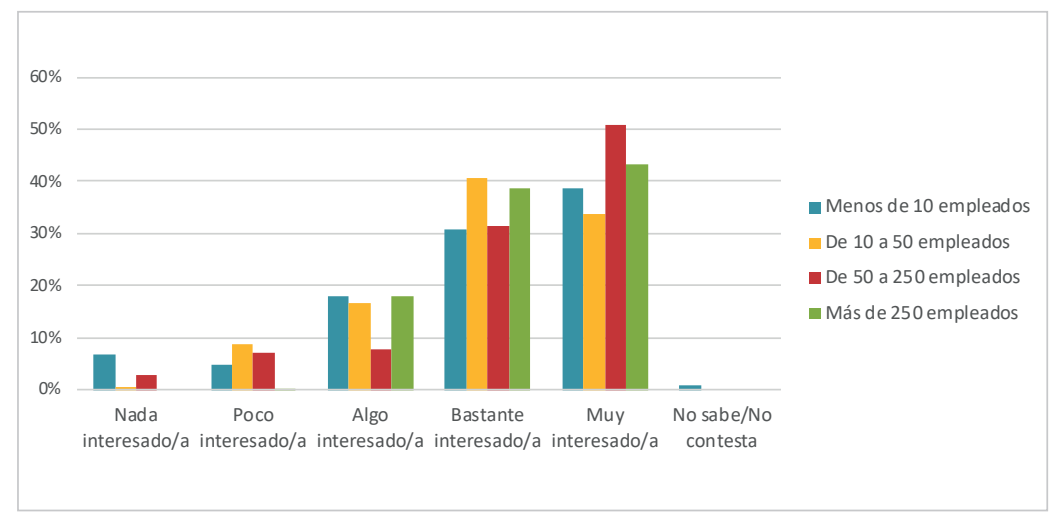

Gráfico 7. ¿Hasta qué punto se considera usted interesado/a en los avances en la ciencia y tecnología aplicados a su sector? Tamaño (datos encuesta CCe).

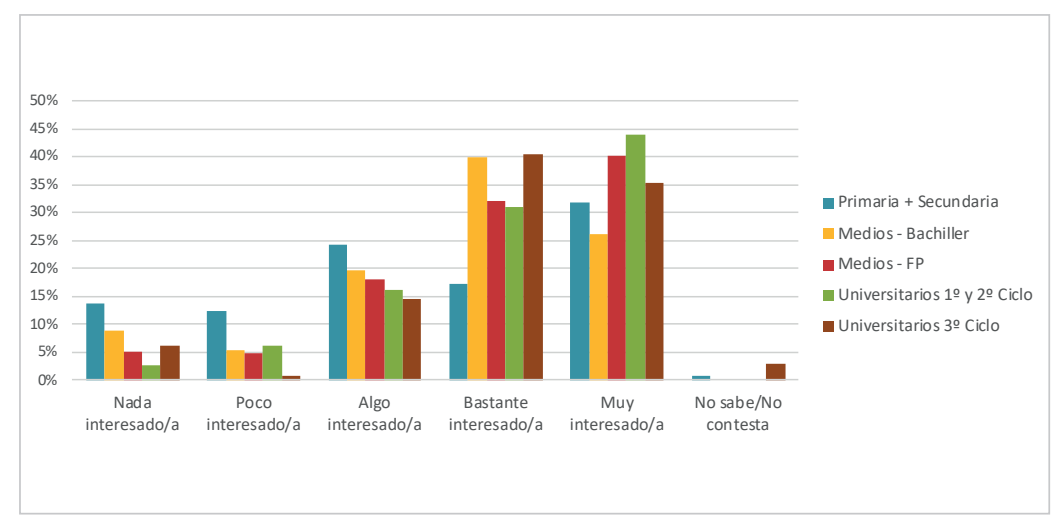

Gráfico 8. ¿Hasta qué punto se considera usted interesado/a en los avances en la ciencia y tecnología aplicados a su sector? Estudios (datos encuesta CCe)

Llegados a este punto destacamos el factor formación y el nivel de estudios cómo determinantes para la implementación de la I+D+i en las empresas como hemos podido constatar a través de la encuesta CCe.

Desde la motivación inicial incide de manera evidente el nivel de estudios. A más alto nivel de estudios mayor interés en los avances de la ciencia y la tecnología. Y es que, mientras el 36,4\% de los encuestados que tienen estudios de nivel secundario (primaria más secundaria) están poco o algo interesados en los avances científico-tecnológicos, el porcentaje va descendiendo hasta el $15,1 \%$ de los encuestados con un nivel de estudios universitarios de tercer ciclo. En sentido contrario, el porcentaje de encuestados que están bastante o muy interesados en los avances de la ciencia y la tecnología y que tienen un nivel de estudios universitarios es muy elevado. El 75,1\% con estudios universitarios de primer y segundo ciclos y el $75,7 \%$ con estudios universitarios de tercer ciclo, lo que indica que a mayor nivel de estudios mayor interés en los avances de la ciencia y tecnología aplicados a su sector, concediendo mayor importancia a la l+D+i para el desarrollo de la propia empresa (gráfico 8).

Esta relación es importante en el cargo que ocupan los encuestados, ya que suele coincidir un mayor nivel de estudios con una más alta responsabilidad en la empresa donde trabajan (tabla 3).

\section{Obstáculos para invertir e implementar la I+D+i en España}

A partir de la encuesta CCe vamos a tener en cuenta cuáles son los principales obstáculos a los que se enfrentan las empresas españolas a la hora de invertir e implementar la I+D+i, analizando las respuestas de los encuestados a la pregunta sobre los beneficios de implementar la I+D+i en su empresa.

Implementar la I+D+i se percibe como un obstáculo en sí mismo al que se enfrentan las empresas, ya que el 38,7\% de los encuestados están bastante o muy de acuerdo con la afirmación que para poder llevar a cabo una correcta implementación de I+D+i hace falta una inversión muy arriesgada con un alto grado de incertidumbre (gráfico 9).

No obstante, esta primera inversión arriesgada que supone un obstáculo para dar el primer paso, luego es vista como bastante o muy beneficiosa a largo plazo por el $77,6 \%$ de los encuestados. Entre los encuestados se percibe que, aunque implementar I+D+i en la empresa supone un obstáculo por la inversión inicial, posteriormente también da a esas empresas una ventaja competitiva, mejorando su desarrollo en el mercado. De hecho, más del $84 \%$ de los encuestados está bastante o muy de acuerdo con estas dos afirmaciones (gráfico 9).

Los encuestados asumen que la inversión que realizan sus empresas para implementar la l+D+i es muy arriesgada y con un alto grado de incertidumbre ya que ellos ven que las ayudas y subvenciones que existen no son apropiadas 
Tabla 3. Cargo en la empresa en relación con sus estudios (datos encuesta CCe)

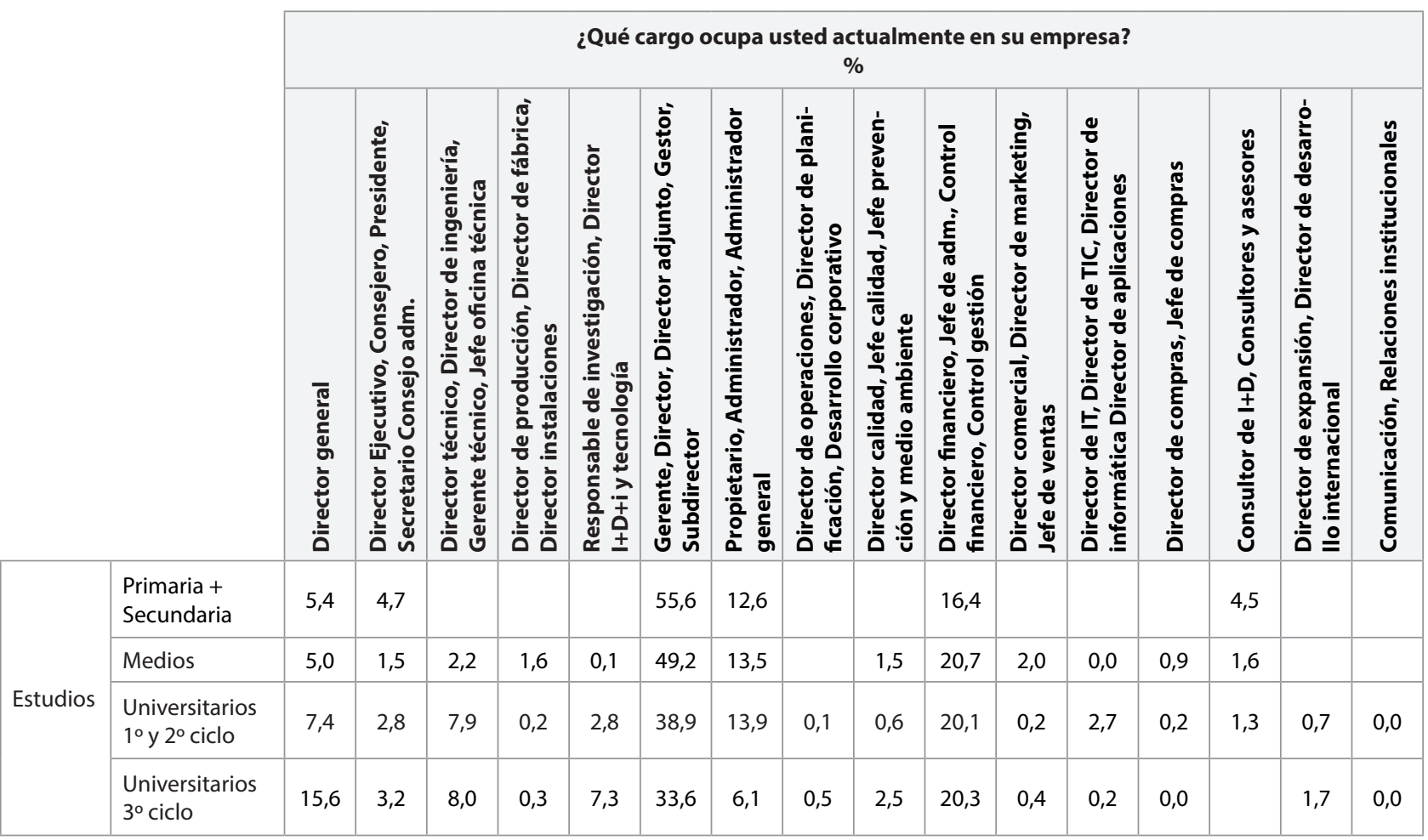

para su empresa. Destacamos además la falta información sobre esas posibles ayudas y subvenciones y el complejo acceso a la financiación.

Otro de los obstáculos a los que se enfrentan las empresas es que el resultado de la inversión revierta en la empresa. Así, a los encuestados se les hizo la siguiente pregunta: La actividad investigadora o innovadora llevada a cabo por su empresa ¿ha dado lugar a la solicitud de patentes o modelos de utilidad?

Los datos de esta pregunta hablan por sí solos, ya que el $81,3 \%$ de las empresas que han hecho alguno de los tipos de I+D+i nunca han iniciado los trámites de solicitud para crear una patente o modelo de utilidad (invención) conforme a la Ley 24/2015, de 24 de julio, de Patentes recogida en el $B O E$ n. 177, de 25 de julio de 2015 (España, 2015) a través de la Oficina Española de Patentes y Marcas (OEPM) (gráfico 10).

Si observamos estos resultados totales por sector empresarial los datos son diferentes. Es decir, el sector empresarial es un factor determinante que afecta a la innovación.

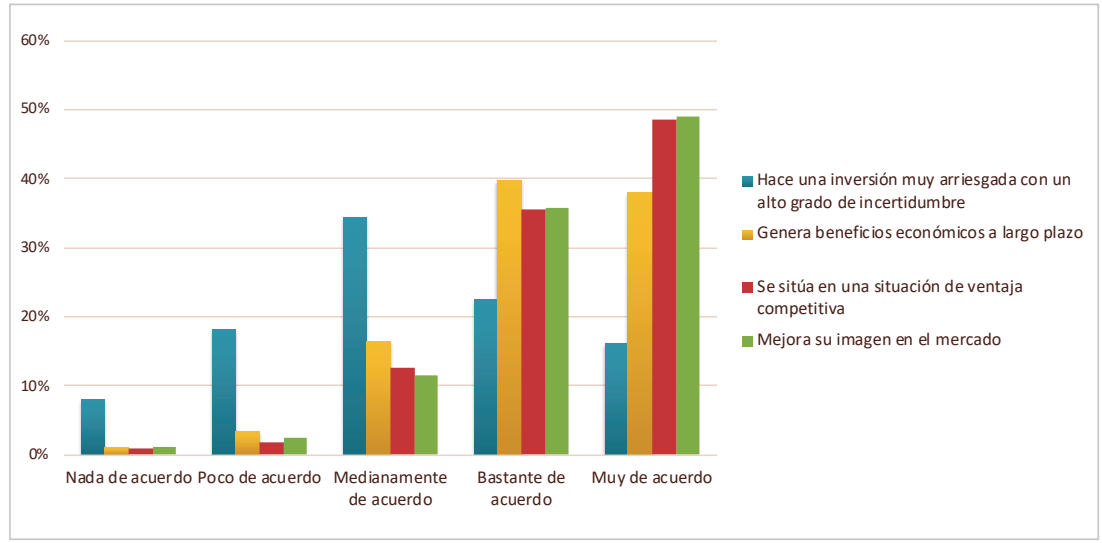

Gráfico 9. Implementar una política de I+D+i... (datos encuesta CCe).

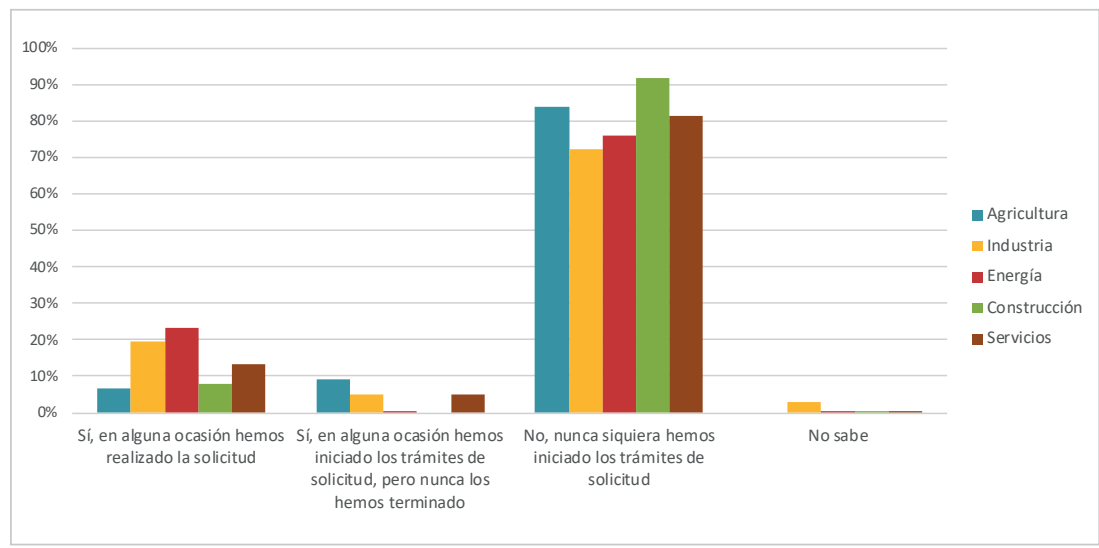

Gráfico 10. La actividad investigadora o innovadora llevada a cabo por su empresa ¿̇ha dado lugar a la solicitud de patentes o modelos de utilidad? Sector (datos encuesta CCe).

En este caso y viendo los resultados por sectores, se observa cómo sí que hay un porcentaje importante de empresas del sector industrial $(19,6 \%)$ y energético $(23,4 \%)$ que han realizado en alguna ocasión una solicitud de patentes o modelos de utilidad frente al 8,0\% del sector de la construcción o al 6,6\% del sector agrícola (gráfico 10). 
Otro de los factores que influyen en la actividad investigadora es el tamaño de la empresa. Aunque las empresas de menos empleados implementen la I+D+i en menor medida que las empresas más grandes, entre las que sí que han realizado algún tipo de $I+D+i$ el porcentaje de las que nunca han iniciado los trámites de solicitud de patentes es de aproximadamente el $80,0 \%$ frente al $67,3 \%$ de las empresas que cuentan con más de 250 empleados (gráfico 11).

Esta pregunta relacionada con las patentes como uno de los resultados finales de la I+D+i tenemos que ponerla en relación con otro obstáculo que encuentran los encuestados a la hora de implementar la I+D+i: problemas derivados del actual sistema de registro de patentes y/o propiedad industrial. Este es un problema importante que encuentran el $22,3 \%$ de los encuestados ${ }^{6}$. Se trata del problema del exceso de burocracia, considerado por casi el $50 \%$ de los encuestados como un obstáculo importante al que se enfrentan las empresas a la hora de invertir e implementar la I+D+i. Asimismo, se hizo a los encuestados una pregunta abierta sobre las barreras u obstáculos que se había encontrado en su empresa a la hora de invertir o implementar la I+D+i. Entre las respuestas más reiteradas podemos destacar las siguientes:

- poco apoyo público,

- coste económico,

- ayudas y subvenciones poco apropiadas para determinadas empresas,

- falta de financiación,

- falta de una estrategia empresarial destinada a la I+D+i,

- rigidez organizativa,

- falta de personal cualificado,

- no hay actitud innovadora,

- escasez de demanda de innovaciones por parte del mercado, y

- falta de información.

\section{Factores que afectan a la innovación}

Como hemos ido haciendo referencia, existen diferentes factores que afectan de alguna manera, ya sea en positivo o negativo al nivel de I+D+i implementado por las empresas.

Uno de estos factores es ver si la empresa que estamos analizando pertenece a un parque científico o tecnológico, un clúster u otra agrupación de empresas cuyo fin sea incentivar la investigación, desarrollo e innovación, ya que estas agrupaciones tienen como objetivo fundamental aumentar la riqueza de la comunidad mediante la promoción de la cultura de la innovación así como la competitividad de las empresas e instituciones asociadas y promocionar la investigación favoreciendo el avance en áreas estratégicas.

Para que se cumplan estos objetivos, un science park estimula y gestiona el flujo de conocimiento y tecnología entre universidades, instituciones de $\mathrm{I}+\mathrm{D}+\mathrm{i}$, empresas y mercados, facilita la creación y el crecimiento de empresas basadas en la innovación a través de procesos de incubación y spin-off, y proporciona otros servicios de valor agregado junto con espacios e instalaciones de alta calidad (IASP, 2018).

Basándonos en esta definición sobre lo que es y lo que implica un parque científico podemos sostener que, si una empresa pertenece a uno, es sinónimo de que conoce los avances científico-tecnológicos de primera mano, y que por tanto se encuentra en mejores condiciones de partida para implementar la I+D+i.

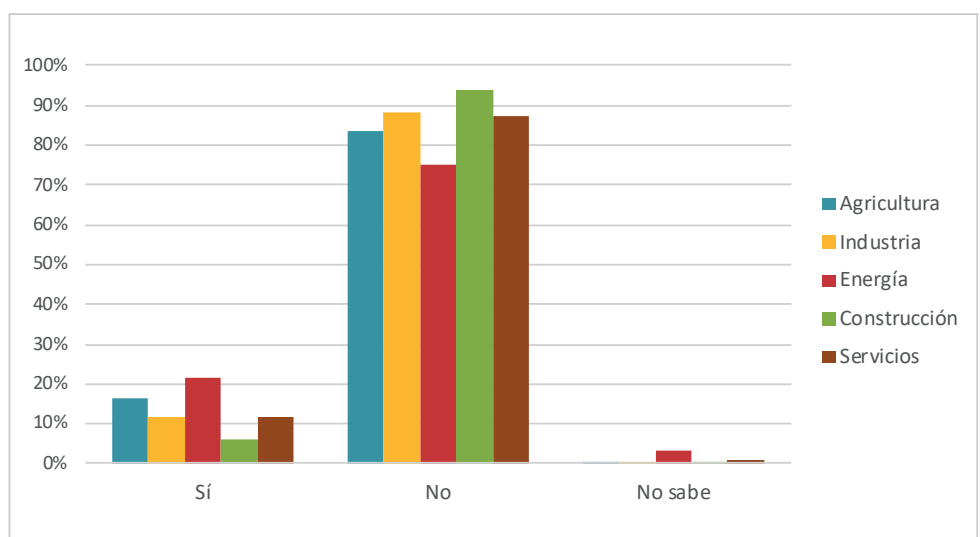

Gráfico 12. ¿Pertenece su empresa a un parque científico o tecnológico, un clúster u otra agrupación de empresas cuyo fin sea incentivar la investigación, desarrollo o innovación? Sector (datos encuesta CCe). 
Este factor tan importante vemos que sólo es cumplido por el 11,1\% de los encuestados, dato muy significativo. Esto implica que casi el $90 \%$ de los encuestados no están trabajando en una empresa asociada a un parque científico.

Sin embargo, este porcentaje será aún más significativo si miramos el sector empresarial en el que están ubicadas estas empresas y el tamaño de las mismas.

El sector es un factor clave que afecta a la I+D+i, ya que mientras los porcentajes de las empresas que pertenecen a un parque científico o tecnológico del sector energético $(21,4 \%)$, la agricultura $(16,6 \%)$ y la industria (11,6\%) son altos, las empresas del sector de la construcción (5,9\%) apenas pertenecen a estos parques científicos (gráfico 12).

Si atendemos al tamaño de la empresa, aunque hay un porcentaje elevado de empresas que no pertenecen a ningún parque científico, el porcentaje de las que sí, va incrementándose en relación con el aumento del número de empleados contratados. Mientras el $10 \%$ de las empresas de menos de 10 empleados pertenece a un parque, las empresas de más de 250 empleados lo están en un 21,2\%.

A través del análisis de la propia encuesta y como factor significativo para la implementación de la I+D+i en España destaca a lo largo de la misma y con componentes tanto cuantitativas como cualitativas el nivel educativo. La educación como motor o desfase de la innovación empresarial. Para hacer esta afirmación nos hemos basado en diferentes preguntas de la encuesta. Entre ellas destacamos la siguiente: ¿ُRecuerda el nombre de alguna institución que se dedique a hacer investigación científica y tecnológica en nuestro país? Sobre la base total de los encuestados percibimos cómo no es mucho mayor el porcentaje de aquellos que responden que no conocen el nombre de alguna institución 59\%, frente a los que han respondido de forma afirmativa $41 \%$ a la cuestión planteada. Por ello, vamos a analizar la pregunta en términos de los estudios que han terminado los encuestados (gráfico 13).

Viendo el gráfico 13 podemos comprender perfectamente por qué el factor educación tiene especial relevancia en cualquier estudio sobre la implementación de la I+D+i en las empresas en una región concreta. Se observa cómo a menor nivel educativo, menor conocimiento de la investigación científica y tecnológica en España: el 13,3\% de los encuestados con nivel de estudios primarios y secundarios conocen alguna de estas institu-

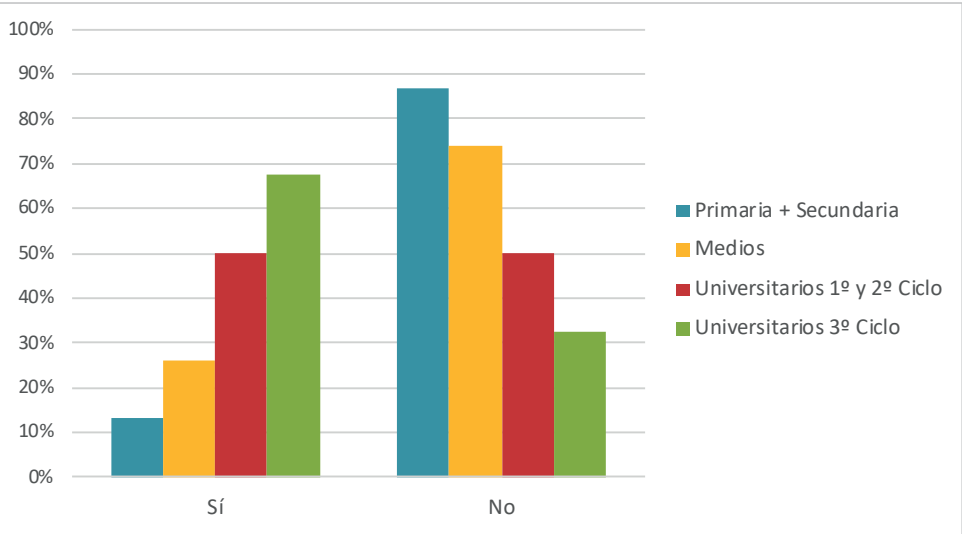

Gráfico 13. ¿Recuerda el nombre de alguna institución que se dedique a hacer investigación científica y tecnológica en nuestro país? Estudios (datos encuesta CCe).

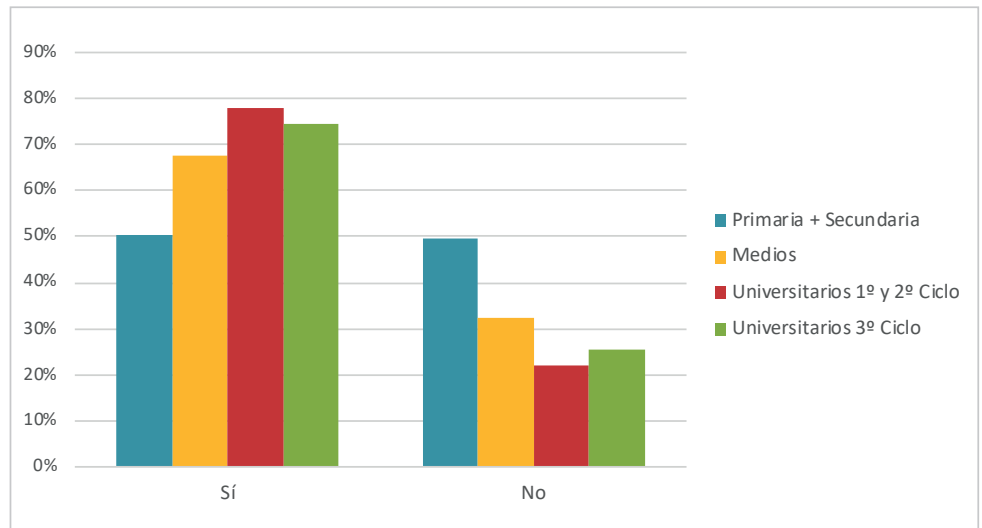

Gráfico 14. ¿Suele informarse para mantenerse al día sobre ciencia y tecnología en su empresa? Estudios (datos encuesta CCe). ciones frente a más de un $50 \%$ de los encuestados con nivel universitario que han respondido afirmativamente a esta misma cuestión (gráfico 13).

Sin embargo, no es esta la única pregunta de la encuesta que refleja el factor educación como uno de los más importantes en un análisis como el que estamos llevando a cabo. Así, cuando se ha preguntado en la encuesta CCe: ¿Suele informarse para mantenerse al día sobre ciencia y tecnología en su empresa?

El $71,2 \%$ de los encuestados ha respondido que sí frente al $28,8 \%$ que no se informa sobre ciencia y tecnología.

Tal y como vimos anteriormente (gráficos 8 y 13), el nivel de estudios es un factor que afecta a la l+D+i, ya que la información sobre ciencia y tecnología y la utilización de fuentes de información relacionadas con este tema como laboratorios e institutos privados $I+D+i$, universidades y organismos públicos de investigación, conversaciones con investigadores, fuentes internas de la empresa, asociaciones profesionales y sectoriales, oficinas de patentes y propiedad industrial, bibliografía o publicaciones científico técnicas..., es mucho mayor cuando el nivel de estudios va aumentando. Es decir, a mayor nivel de estudios más información y utilización de fuentes bibliográficas específicas sobre ciencia y tecnología (gráfico 14).

Estos datos están en paralelo con el informe del INE que nos muestra dos claras consideraciones: 
"la de la permanencia de un destacado colectivo de empresas (casi un 30\%) que consideran que la innovación no es precisa, puesto que no se demanda, y la del alza de las empresas que reconocen en los déficits del conocimiento uno de los factores que obstaculiza la adopción de la innovación" (González-Hermoso-de-Mendoza; Sánchez-López, 2010, p. 254).

\section{Resultados destacados de la encuesta CCe sobre la innovación en España}

La investigación, desarrollo e innovación son asuntos comunes y determinantes para el funcionamiento y desarrollo de una empresa. Sin embargo y a través de los resultados obtenidos en la encuesta advertimos cómo la I+D+i no está implementada en todas las empresas españolas con el mismo grado. Hemos rastreado a través de la misma dónde radican estas diferencias.

Tradicionalmente la innovación ha estado ligada a un contexto exclusivamente industrial y tecnológico. Y es que hay que recordar que el Manual de Oslo (OCDE, 2006) en su primera edición sólo consideraba como tal la innovación industrial. Fue en las sucesivas versiones cuando se amplió el concepto de innovación a un mayor número de empresas centrándose en productos y procesos para finalmente incorporar el sector servicios. De este modo, la idea tradicional de innovación sigue estado presente, aunque ya en menor medida, puesto que los sectores empresariales cada vez están más diversificados y cada empresa, independientemente del sector en que se encuadre, reconoce que la innovación es importante para su futuro empresarial. El actual tejido empresarial comprende los siguientes sectores analizados:

- agricultura,

- industria,

- energía,

- construcción, y

- servicios,

siendo los sectores industrial y energético los más innovadores hasta la fecha. Sin embargo, esto está cambiando ya que, aunque es verdad que el $47,8 \%$ de los encuestados piense que es bastante difícil hacer I+D en España y el $91,6 \%$ de los mismos no dispone de un departamento propio de investigación e innovación en su empresa, el $85 \%$ del total de entrevistados cree que la I+D+i es bastante o muy útil.

A la hora de implementar una política de innovación con resultados favorables, los sectores industrial, energético y agrario son los que más fácil lo tienen ya que son los sectores más interesados en los avances en la ciencia y tecnología aplicados a su sector. Si ponemos el foco de atención en los resultados de la encuesta CCe observamos cómo los encuestados han respondido de forma distinta a la pregunta: ¿Hasta qué punto se considera usted interesado/a en los avances en la ciencia y tecnología aplicados a su sector? Así destaca que los encuestados del sector energético están bastante o muy interesados en un $93,4 \%$; el sector agrario en un $81,2 \%$ y el sector industrial en un $70,3 \%$.

Aunque estos sectores son los que a priori implementan una política de innovación en sus empresas, también hay que considerar otro factor importante: la educación. Si atendemos al nivel de educación de los encuestados vemos cómo a mayor nivel de estudios más conocimiento se tiene acerca del valor de la innovación empresarial. Este hecho se repite independientemente del sector en el que se encuadre la empresa, así como del tamaño de las mismas medido en número de empleados. El nivel educativo es fundamental para poder llevar a cabo una política de innovación empresarial, ya que cuanto más conocimiento específico sobre la cultura innovadora, más posibilidades de implementar la innovación lo que se traduce en crecimiento, productividad competitividad y permanencia en el mercado.

El consumo de información y documentación especializada sobre ciencia y tecnología por parte de los empresarios muestra cómo la innovación va unida a la información. La educación y el nivel de estudios influyen significativamente en este aspecto. El nivel de estudios afecta de manera determinante a la l+D+i empresarial y cómo la desinformación sobre ciencia y tecnología lo observamos en el apartado de la no utilización de fuentes de información. Formación e innovación también se conjugan conjuntamente en las políticas de I+D+i empresarial. Y es que como advierte Ferrer-Sapena

"la búsqueda en bases de datos de prestigio científico-técnicas y de patentes tiene la gran ventaja de asegurar un cierto grado mínimo de calidad en cuanto los documentos que han sido recogidos en ellas" (Ferrer-Sapena, 2008).

Más del 70\% de los encuestados han afirmado que se informan para mantenerse al día sobre ciencia y tecnología en su empresa. Este porcentaje se pude escalar atendiendo al nivel de estudios de los encuestados, ya que volvemos otra vez a insistir en que a mayor nivel de estudios menos desinformación científica y tecnológica.

Siguiendo con la afirmación de Ferrer-Sapena (2008) constatamos la importancia dada a las patentes, ya que mediante las patentes registradas por las empresas podemos conocer la evolución de la innovación de las y, además, podemos hacer análisis comparativos a través del registro de patentes en base a la vigilancia tecnológica e inteligencia competitiva.

La vigilancia tecnológica es un elemento clave en la política de I+D+i, ya que permite a las empresas captar información del exterior y de la propia empresa sobre ciencia y tecnología para posteriormente convertirla en conocimiento para tomar decisiones con un menor riesgo sobre la materia (Aenor, 2018), pero también les permite identificar a las empresas que cuentan con amplios avances y desarrollos tecnológicos que harán que la empresa pueda beneficiarse de ellos 
minimizando así sus inversiones en I+D+i mediante la implementación de una política de benchmarking empresarial (Escorsa-Castells; Maspons-Bosch, 2001; Escorsa-Castells; Valls-Pasola, 2003).

Sin embargo, y como hemos destacado en el apartado 4. "Obstáculos para invertir e implementar la I+D+i", la actividad investigadora o innovadora que llevan a cabo las empresas no siempre suele dar lugar a solicitudes de patentes o modelos de utilidad, siendo el análisis de patentes uno de los indicadores de resultados científicos y tecnológicos que miden la ciencia y tecnología de un país. De hecho, un $81,3 \%$ de las empresas nunca ha iniciado los trámites de solicitud de una patente. Este porcentaje se reduce si atendemos por sectores empresariales, ya que el $23,4 \%$ de las empresas del sector energético y el $19,6 \%$ del sector industrial sí que han realizado esta solicitud de patentes ante la Oficina Española de Patentes y Marcas (OEPM).

"El bajo número de patentes y la escasa presencia en publicaciones internacionales es un claro reflejo, por una parte, de una capacidad de absorción insuficiente y, por otra, de la poca madurez en el mercado internacional de nuestro tejido productivo" (Rojo; Gómez, 2006).

Como resultado del análisis de los datos de la encuesta CCe se percibe que uno de los principales obstáculos a los que se enfrentan las empresas en relación con la innovación es el sistema de registro de patentes y propiedad intelectual, ya que sólo el $21,6 \%$ de los encuestados ha respondido que no encuentran ningún problema en el sistema de registro, dato que contrasta con el alto porcentaje de encuestados $(40,4 \%)$ que no han querido contestar a la pregunta. Podríamos volver a concluir aquí que el desconocimiento sobre el sistema de patentes por parte de la empresa española está directamente relacionado con la formación. Una de las barreras percibidas por tanto con el uso del registro de patentes está asociado al exceso de burocracia, considerado por casi el 50\% de los encuestados como un obstáculo importante al que se enfrentan las empresas a la hora de invertir e implementar la I+D+i.

Lo cierto es que el tamaño de la empresa o número de empleados contratados influye significativamente a la hora de implementar la innovación en las empresas, puesto que las empresas que cuentan con un mínimo de empleados apenas implementan la I+D+i frente a las grandes empresas con un número de empleados mayor a 250 que sí que llevan a cabo una inversión importante para poder realizar innovación y conseguir tanto patentes como modelos de utilidad. Podemos igualmente concluir que las pymes en España, que constituyen un porcentaje mayoritario del sistema productivo tienen, perciben serias barreras frente a la implementación de la I+D+i.

Enlazamos otra vez con la encuesta, pues otra de las barreras u obstáculos a los que se enfrentan las empresas a la hora de invertir en innovación es el coste económico que supone la política de I+D+i. Y es que, aunque casi el $80 \%$ de los encuestados reconocen que una estrategia empresarial destinada a la l+D+i genera beneficios económicos a largo plazo, la inversión inicial posee un alto grado de incertidumbre para llevar a cabo esta política de innovación por lo que es vista con recelo por el $73,1 \%$ de los encuestados. De esta forma, las empresas de menos empleados no suelen implementar la innovación porque esta inversión inicial les supone un riesgo mucho mayor que para las empresas que cuentan con un número de empleados más elevado.

En el trabajo de Uyarra (2006), financiado por Naciones Unidas y la Universidad de Maastricht, se define la necesidad de cooperar entre el sector público y privado para alcanzar logros en las inversiones de I+D+i denominándola como policy mix for R\&D siendo ésta la combinación de instrumentos de políticas que interactúan para influir en la cantidad y calidad de las inversiones en I+D en los sectores público y privado. Es por ello que las fuentes de financiación son importantes,

"resultando obligado que la innovación esté cada vez más presente en las políticas de los gobiernos nacional y autonómicos de nuestro país" (Ferrer-Sapena, 2008).

Debemos apuntar que esto no es un problema exclusivamente español, y es recogido en la investigación realizada en Holanda por Faber, Van-Dijk y Van-Rijnsoever:

"Dado que la participación de las pequeñas y medianas empresas (pymes) en los programas europeos de investigación colaborativa es menor de lo que se había esperado, este estudio investiga los motivos de las pymes basadas en I+D para la (no) participación en estos programas. Encontramos que los programas europeos de investigación colaborativa atraen la participación de un número bastante limitado de pymes especialmente basadas en las que tienen experiencia previa con la colaboración internacional, en los incentivos del costo compartido y el intercambio de conocimiento y la barrera formada por los costos de participación en estos programas. Se derivan medidas de política que podrían mejorar la participación de las pymes en los programas europeos de investigación colaborativa derivadas de nuestros resultados" (Faber; Van-Dijk; Van-Rijnsoever, 2015).

Las fuentes de financiación tanto públicas como privadas ${ }^{8}$ son importantes, ya que la inversión de las empresas en innovación siempre generará efectos positivos tanto en la propia empresa a través de su productividad y competitividad, independientemente del sector en el que se encuadre, como en el sistema económico y social. Es revelador el dato apuntado por la OCDE sobre el fracaso de las empresas españolas:

"Sólo el 29\% de las empresas españolas sobrevive al quinto año. En el primer año de creación, el 74\% de las empresas españolas fracasa. En el primer año de creación, las empresas españolas también están a la cabeza de las que más fracasan en Europa, con un 74\%. En el segundo año, el porcentaje se reduce al 60\%; en el tercero, al $45 \%$ y en el cuarto, al 6\%" (OCDE, 2015a). 
Pero hay elementos para ser positivos. Una de las ideas siempre presente para mejorar la innovación de las empresas es hacer efectiva la demandada alianza empresa-universidad. El desarrollo de las spin-offs en los campus universitarios en España, está permitiendo la creación de las empresas más innovadoras hoy.

"La creación de empresas de base tecnológica en el seno de las universidades, especialmente las spin-offs universitarias, se ha convertido en los últimos años en uno de los mecanismos más eficaces de transferencia de resultados de investigación y tecnología desde los centros de investigación universitarios al sector productivo. Como consecuencia, este tipo de empresas está siendo motivo de estudio por el importante papel que se les atribuye en los procesos de innovación, la generación de empleo cualificado y, por tanto, su aportación al desarrollo socioeconómico" (Iglesias-Sánchez; Jambrino-Maldonado; Peñafiel-Velasco, 2012).

El trabajo de Lacy et al. pone de manifiesto este aspecto para Estados Unidos

"Las alianzas entre universidades e industrias de los Estados Unidos han existido durante varias décadas y en los últimos años se han vuelto generalmente más variadas, de alcance más amplio, más agresivas, experimentales y de mayor visibilidad pública. Además, en las últimas décadas, los intereses públicos y privados han abogado por políticas y leyes gubernamentales para promover globalmente la comercialización de la ciencia universitaria" (Lacy et al., 2014).

Esta es una de las ideas con los que nos gustaría concluir, el desarrollo de la innovación en los clusters universitarios está ayudando a cambiar la percepción de la innovación y en general de la cultura científica en las empresas españolas. Esperemos ver pronto resultados en la economía y por tanto en el empleo.

El informe de resultados de la innovación en Europa publicado en 2017 con datos de 2016, Innovation ouput indicator 2017 (Vértesy, 2017), mide cómo las ideas son capaces o no de llegar al mercado dependiendo de una serie de componentes. Se basa en cuatro componentes:

- la innovación tecnológica;

- el empleo;

- la competitividad;

- la inversión en bienes y servicios intensivos en conocimiento en empresas, dentro de los sectores más innovadores.
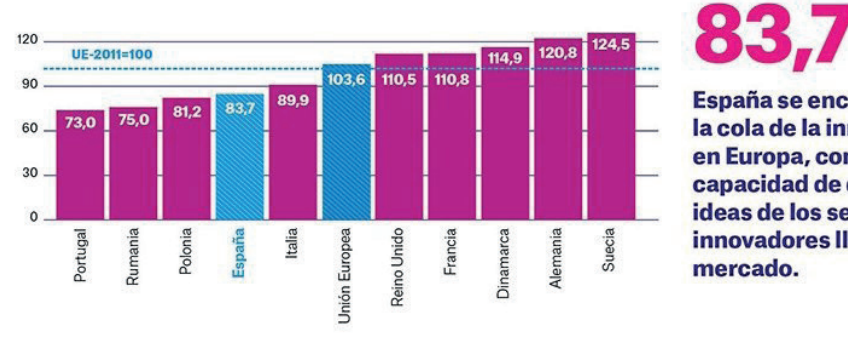

España se encuentra a la cola de la innovación en Europa, con escasa capacidad de que las ideas de los sectores innovadores lleguen al mercado.

Sobre estos datos el Observatorio Social de

Gráfico 15. Innovation output indicator 2014.

Fuentes: Joint Research Centre y La Caixa.

https://observatoriosociallacaixa.org/es/-/innovation-output-indicator-2014-

La Caixa, elabora unos informes poniendo el foco en España. Según dicho observatorio con datos publicados en 2016, España,

"está a la cola de la UE en eficiencia de sectores I+D. El país tiene 83,7 puntos en el Indicador de Resultados de la Innovación, una valoración muy alejada de la media regional, de 103,6 puntos" (Observatorio Social de La Caixa, 2016).

\section{Conclusiones y discusión sobre la innovación en España a partir de los resultados de la encuesta $\mathrm{CCe}$}

El objetivo de este trabajo ha sido presentar, a la luz del proyecto Cultura científica empresarial, un análisis del vínculo que existe entre educación, innovación y competitividad (Osuna-Alarcón; Rey-Rocha; López-Navarro, 2018) y por tanto, la importancia de la educación y del uso de las fuentes de datos para el desarrollo socio-económico de España que permita dirigirnos hacia un nuevo modelo productivo más tecnológico y competitivo, donde prime la innovación.

El éxito y en muchos casos la supervivencia de una empresa tiene que ver con el esfuerzo que dedica a la innovación, la competitividad, la eficacia y la gestión. Como hemos visto por los datos extraídos de la encuesta CCe, mejorar la estrategia comparativa en estos ámbitos por parte de las empresas españolas se hace imprescindible.

Resulta ineludible, por tanto, que la innovación en España se desarrolle, consiguiendo así mejorar uno de los pilares de la permanencia de las empresas en el mercado. En este esfuerzo de extrapolar los datos de una encuesta hemos tenido muy en cuenta en primer lugar los datos de la propia encuesta CCe, pero también la Encuesta de percepción social de la ciencia y la tecnología de Fecyt, estudio que desde 2002 recoge cada dos años el análisis y los resultados de la Encuesta de percepción social de la ciencia y la tecnología en España (Fecyt, 2018); y la Encuesta sobre innovación en las empresas del INE que tiene como objetivo, ofrecer información directa sobre el proceso de innovación en las empresas (INE, 2019).

Por otra parte, ha sido relevante la opinión de otros actores implicados como es la Cámara de Comercio de España quien declara que la competitividad para ser efectiva debe sustentarse en la innovación. Así como alguno de los responsables de la financiación de las empresas como es La Caixa. 
También hemos intentado insertar los datos para ver la comparativa en un entorno regional como es el europeo. En un esfuerzo por reducir la brecha entre ciencia y sociedad, la Comisión Europea impulsó el concepto de Investigación e Innovación Responsable $(R R I)$, un tema transversal dentro de Horizonte $2020^{9}$. El enfoque de toda Europa busca poner los asuntos de I+D+i en primer plano, anticipar sus consecuencias e involucrar a la sociedad en la discusión sobre cómo la ciencia y la tecnología pueden ayudar a dar forma al mundo del mañana. Se necesitan herramientas concretas para implementar $R R I$ en toda Europa y realizar esta visión.

A través de la encuesta CCe hemos querido reseñar la necesidad de estudiar el vínculo ciencia-empresa como relación social para dejar constancia de que la innovación en la empresa española es escasa. Más significativa en las grandes empresas e insuficiente en las pymes para su propia supervivencia, siendo éstas la base del tejido productivo español.

Por tanto, estamos de acuerdo con la siguiente manifestación

“existe una escasa participación empresarial en la financiación y ejecución de la I+D+i por la falta de estrategias para la apropiación por parte de las empresas del conocimiento generado por universidades y centros públicos de investigación" (Fernández-Zubieta et al., 2016; OCDE, 2015b).

Destacando que la innovación es lo que permite a la empresa su supervivencia en un mercado tecnológico y por tanto cambiante, parece lógico que si entre las políticas gubernamentales españolas está mejorar las tasas y la calidad del empleo se deben crear mecanismos gubernamentales que ayuden y apuesten decididamente por la innovación en las empresas ya que estas por sí mismas, como vemos por el análisis de la encuesta CCe, no lo han hecho suficientemente hasta la fecha.

Las barreras que encuentran las empresas a la hora de innovar son de formación, burocráticas, de acceso a la información y de incertidumbre ante el cambio, además de financiación. El desarrollo de políticas públicas de apoyo decidido a la innovación en las empresas unido a la introducción de la innovación como materia en el sistema educativo tendría consecuencias favorables para la economía, el empleo y el desarrollo de las pymes, podríamos decir incluso que para su subsistencia ya que actualmente el $74 \%$ de las mismas no llegan a cumplir un año de vida (OCDE, 2015b). Así mismo señalamos que la innovación en los clusters universitarios está ayudando a cambiar la percepción de la innovación y en general de la cultura científica en las empresas españolas. Como podemos observar, el puesto que ocupa España en el ranking del Índice mundial de innovación de $2019^{10}$ (Dutta;

Tabla 4. Índice mundial de innovación de España (datos OMPI)

\begin{tabular}{|l|c|c|}
\hline Fecha & Ranking de innovación & Índice de innovación \\
\hline 2019 & $29^{\circ}$ & 47,85 \\
\hline 2018 & $28^{\circ}$ & 48,68 \\
\hline 2017 & $28^{\circ}$ & 48,80 \\
\hline 2016 & $28^{\circ}$ & 49,19 \\
\hline 2015 & $27^{\circ}$ & 49,07 \\
\hline 2014 & $27^{\circ}$ & 49,27 \\
\hline 2013 & $26^{\circ}$ & 49,41 \\
\hline 2012 & $29^{\circ}$ & 47,20 \\
\hline 2011 & $32^{\circ}$ & 43,81 \\
\hline
\end{tabular}

Lanvin; Wunsch-Vincent, 2019) es el 29 de 129 países, con una puntuación de 47,85 sobre 100, por suerte lejos del índice 43,81 que obtuvo en 2011. Destacamos que en el ranking de los 3 países con mayor Índice mundial de innovación se encuentran: Suiza con una puntuación de 67,24; Suecia con 63,65 y Estados Unidos con 61,73 sobre 100.

Ser o no ser competitivo en términos de innovación resume las opciones de supervivencia y triunfo o fracaso de las empresas. Debemos ser capaces de ver la I+D+i como una ventaja competitiva (Chesnais, 1986; Wilson, 2003; Cornejo-Cañamares, 2009) dentro de la cultura científica empresarial, sobre la base de una recuperación económica a medio y largo plazo (Comisión Europea, 2010a; 2010c).

Por suerte el año 2016 fue el segundo consecutivo de subida de la inversión en I+D en España después de seis años de contracción entre 2009 y 2014 (Cotec, 2018), ya que el gasto en innovación tecnológica se incrementó un 1,3\% en 2016 y se situó en 13.857 millones de euros (INE, 2019).

\section{Notas}

1. Estudio financiado por el Ministerio de Economía y Competitividad a través del Plan Estatal de Investigación Científica y Técnica y de Innovación. Proyecto de investigación Cultura científica, percepción y actitudes ante la ciencia y la innovación en el sector empresarial español (CSO2014-53293-R) de la Agencia Estatal Consejo Superior de Investigaciones Científicas (CSIC).

2. La encuesta CCe (Cultura científica, percepción y actitudes ante la ciencia y la innovación en el sector empresarial español) se puede consultar en las siguientes direcciones:

http://digital.csic.es/handle/10261/171841 http://digital.csic.es/handle/10261/177927

3. El Proyecto CCe ha dividido la encuesta en los siguientes sectores económicos: agricultura, industria, energía, construcción y servicios (incluye también transporte, comercio y hoteles), según la agrupación de la Clasificación nacional de actividades económicas (CNAE).

También hay que hacer constar que las respuestas de los empleados de estos sectores económicos se han estructurado atendiendo al número de empleados con los que cuenta cada una de estas empresas:

- micro empresa: hasta 9 empleados;

- pequeña empresa: de 10 a 49 empleados; 
- mediana empresa: de 50 a 249 empleados;

- gran empresa: de 250 o más empleados.

4. Los cargos directivos de las empresas se han diversificado y han adoptado un cambio significativo en relación con sus funciones a través de diferentes "etiquetas". No es de extrañar, por tanto, que actualmente tratemos con neologismos de los cargos de las organizaciones empresariales:

- CEO (Chief executive officer) o Director ejecutivo.

- CMO (Chief marketing officer) o Director de marketing.

- CCO (Chief communications officer) o Director de comunicación.

- CTO (Chief technology officer) o Director del área tecnológica.

- CIO (Chief information officer) o Director de información.

- CFO (Chief financial officer) o Director financiero.

- COO (Chief operating officer) o Director de operaciones.

- SCM (Supply chain manager) o Gerente de compras.

- CHRO (Chief human resources officer) o Director de recursos humanos.

5. Oficina Española de Patentes y Marcas:

https://www.oepm.es/es/index.html

6. El registro de los derechos de propiedad industrial no es obligatorio, pero sí recomendable, ya que el nacimiento del derecho exclusivo sobre los mismos nace del registro válidamente efectuado.

7. Para conocer mejor la división de las fuentes generales de información acudir a Muñoz-Cañavate (2003).

8. El sistema español de innovación fue modelado en el libro blanco El sistema español de innovación: diagnósticos y recomendaciones (Cotec, 1998), donde se han identificado cinco subsistemas:

- administración pública;

- sistema público de I+D;

- infraestructuras de soporte a la innovación;

- empresas;

- entorno.

9. Entre los objetivos de Horizonte 2020 se encuentra hacer de Europa un lugar más atractivo para invertir en la investigación y la innovación, fomentar la industria altamente competitiva, impulsar la innovación en pequeñas y medianas empresas, invertir en tecnologías clave para el desarrollo industrial...

10. El Índice mundial de innovación clasifica los resultados de la innovación de unos 130 países y economías con más de 80 indicadores. El informe, publicado conjuntamente por la OMPI, la Cornell University y el Insead, ofrece una clasificación anual de las capacidades y el desempeño de las economías de todo el mundo en innovación (Dutta; Lanvin; Wunsch-Vincent, 2019).

\section{Referencias}

Aenor (2018). UNE 166006:2018. Gestión de la I+D+i: Sistema de vigilancia e inteligencia. https://www.aenor.com/normas-y-libros/buscador-de-normas/UNE?c=N0059973

Cámara de Comercio de España (2017). Innovación, digitalización y competitividad. https://www.camara.es/innovacion-y-competitividad/como-ser-competitivo

CCe. Cultura científica empresarial (2017). Proyecto de investigación: Cultura científica, percepción y actitudes ante la ciencia y la innovación en el sector empresarial español. Proyecto de Investigación. Ministerio de Economía, Industria y Competitividad. http://www.culturacientificaempresarial.es

Chesnais, François (1986). "Science, technologie et compétitivité". STI Science technologie industrie revue, n. 1.

Comisión Europea (2005a). Special Eurobarometer 224: Europeans, science and technology. Bruselas: Comisión Europea. https://data.europa.eu/euodp/es/data/dataset/S447_63_1_EBS224

Comisión Europea (2005b). Special Eurobarometer 225: Social values, science \& technology. Bruselas: Comisión Europea. http://data.europa.eu/euodp/en/data/dataset/S448_63_1_EBS225

Comisión Europea (2007). Special Eurobarometer 282: Scientific research in the media. Bruselas: Comisión Europea. https://data.europa.eu/euodp/es/data/dataset/S616_67_2_EBS282

Comisión Europea (2010a). Iniciativa emblemática de Europa 2020: Unión por la innovación. SEC(2010) 1161. COM(2010) 0546 final, 06/10/2010. Bruselas: Comisión Europea.

https://eur-lex.europa.eu/legal-content/ES/TXT/?qid=1567421891307\&uri=CELEX:52010DC0546

Comisión Europea (2010b). Special Eurobarometer 340: Science and technology. Bruselas: Comisión Europea. https://data.europa.eu/euodp/es/data/dataset/S806_73_1_EBS340 
Comisión Europea (2010c). Europe 2020 Flagship initiative innovation union. Luxembourg: Publications Office of the European Union. ISBN: 9789279176883

https://doi.org/10.2777/27497

Cornejo-Cañamares, María (2009). La cultura de innovación. Informes técnicos Ciemat, n. 1169. Madrid: Centro de Investigaciones Energéticas, Medioambientales y Tecnológicas.

http://rdgroups.ciemat.es/documents/69177/122473/M_Cornejo_1169.pdf/8bd39959-686e-4c87-ab72-23eebee00aaa

Cotec (1998). El sistema español de innovación. Diagnóstico y recomendaciones: Libro blanco. Madrid: Fundación Cotec para la Innovación Tecnológica. ISBN: 849227204 X

http://personales.upv.es/igil/libro_blanco.pdf

Cotec (2018). Informe Cotec 2018. Madrid: Fundación Cotec para la Innovación Tecnológica. ISBN: 9788492933396 http://informecotec.es/media/Informe-Cotec_2018_versi\%C3\%B3nweb.pdf

Dutta, Soumitra; Lanvin, Bruno; Wunsch-Vincent, Sacha (eds.) (2019). Global innovation index 2019: Creating healthy lives. The future of medical innovation. Cornell University; Insead; OMPI. ISBN: 9791095870142

https://www.wipo.int/edocs/pubdocs/en/wipo_pub_gii_2019.pdf

Einsiedel, Erling (2000). “Understanding 'publics' in the public understanding of science”. In: Dierkes, Meinolf; Von-Grote, Claudia (eds.). Between understanding and trust: The public, science and technology. Amsterdam: Harwood, pp. 205216. ISBN: 9780415516211

Eizaguirre, Andoni (2009). "Los estudios sobre percepción social de la ciencia”. Acciones e investigaciones sociales, n. 27, pp. 23-53.

https://doi.org/10.26754/ojs_ais/ais.200927342

Escorsa-Castells, Pere; Maspons-Bosch, Ramon (2001). De la vigilancia tecnológica a la inteligencia competitiva. Madrid: Prentice- Hall. ISBN: 8420530573

Escorsa-Castells, Pere; Valls-Pasola, Jaume (2003). Tecnología e innovación en la empresa. Barcelona: Universidad Politécnica de Cataluña. ISBN: 8483017067

España (2015). “Ley 24/2015, de 24 de julio, de patentes". BOE, n. 177, 25 julio.

https://www.boe.es/eli/es/l/2015/07/24/24/con

Faber, Jan; Van-Dijk, Jaco; Van-Rijnsoever, Frank (2015). “Incentives and barriers for R\&D-based SMEs to participate in European research programs: An empirical assessment for The Netherlands". Science and public policy, v. 43, n. 3, pp. 414-428.

https://doi.org/10.1093/scipol/scv050

Fecyt (2007). Apuntes sobre los estudios de percepción social de la ciencia y de la tecnología. Madrid: Fundación Española para la Ciencia y la Tecnología.

https://www.upf.edu/pcstacademy/_docs/ApuntesFecyt.pdf

Fecyt (2018). Percepción social de la ciencia y la tecnología en España. Madrid: Fundación Española para la Ciencia y la Tecnología.

https://icono.fecyt.es/informes-y-publicaciones/percepcion-social-de-la-ciencia-y-la-tecnologia-en-espana

Fernández-Zubieta, Ana; Andújar-Nagore, Inés; Giachi, Sandro; Fernández-Esquinas, Manuel (2016). “New organizational arrangement for public-private research collaboration". Journal of the knowledge economy, v. 7, n. 1, pp. 80-103. https://doi.org/10.1007/s13132-015-0292-1

Ferrer-Sapena, Antonia (2008). “Información en la empresa para innovar y competir". El profesional de la información, v. 17 , n. 5 , pp. 481-486.

https://doi.org/10.3145/epi.2008.sep.01

González-Hermoso-de-Mendoza, Alfonso; Sánchez-López, Antonio J. (2010). "La cultura innovadora en la empresa". Mediterráneo Económico. Innovación y desarrollo económico, v. 17, pp. 245-260. Almería: Publicaciones Cajamar. https://dialnet.unirioja.es/servlet/articulo?codigo $=4754539$

Gouardères, Frédéric (2019). "Política de innovación". Fichas técnicas sobre la Unión Europea. Bruselas: Parlamento Europeo.

http://www.europarl.europa.eu/factsheets/es/sheet/67/innovation-policy

Grupo de Investigación en Evaluación y Transferencia Científica (2017). Cultura científica, percepción y actitudes ante la ciencia y la innovación en el sector empresarial español. Madrid: Centro de Ciencias Humanas y Sociales; Consejo Superior de Investigaciones Científicas.

http://investigacion.cchs.csic.es/etc/es/content/cultura-cient\%C3\%ADfica-percepci\%C3\%B3n-y-actitudes-ante-laciencia-y-la-innovaci\%C3\%B3n-en-el-sector-empre 
IASP (2018). Definitions. International Association of Science Parks and Areas of Innovation.

https://www.iasp.ws/our-industry/definitions

Iglesias-Sánchez, Patricia P.; Jambrino-Maldonado, Carmen; Peñafiel-Velasco, Antonio (2012). “Caracterización de las spin-off universitarias como mecanismo de transferencia de tecnología a través de un análisis clúster". Revista europea de dirección y economía de la empresa, v. 21, n. 3, pp. 240-254.

https://doi.org/10.1016/j.redee.2012.05.004

INE (2019). Encuesta sobre innovación en las empresas. Madrid: Instituto Nacional de Estadística. http://www.ine.es/dyngs/INEbase/es/operacion.htm?c=Estadistica_C\&cid=1254736176755\&menu=ultiDatos\&idp=1254735576669

Lacy, William B.; Glenna, Leland L.; Biscotti, Dina; Welsh, Rick; Clancy, Kate (2014). "The two cultures of science: Implications for university-industry relationships in the U.S. agriculture biotechnology". Journal of integrative agriculture, v. 13, n. 2, pp. 455-466.

https://doi.org/10.1016/S2095-3119(13)60667-X

López-Navarro, Irene; Rey-Rocha, Jesús; González-Bravo, María-Isabel; Osuna-Alarcón, María-Rosario; Muñoz-Pérez, Félix; Fernández-de-Pinedo-Echevarría, Nadia; Sáiz-González, Patricio; Cuesta-Santianes, María-José; Castro-Balaguer, Rafael (2016). "Percepción de la ciencia en el sector empresarial español”. En: XII Congreso español de sociología: Grandes transformaciones sociales, nuevos desafíos para la sociología.

http://fes-sociologia.com/percepcion-de-la-ciencia-en-el-sector-empresarial-espanol/congress-papers/2311

Ministerio de Ciencia, Innovación y Universidades (2019a). Horizonte 2020.

https://eshorizonte2020.es

Ministerio de Ciencia, Innovación y Universidades (2019b). Sistema español de Ciencia, Tecnología e Innovación. http://bit.ly/3ciwBvt

Muñoz-Cañavate, Antonio (2003). "Sistemas de información en las empresas". Hipertext.net, n. 1. https://www.upf.edu/hipertextnet/numero-1/sistem_infor.html

Observatorio Social de La Caixa (2016). “Indicador de resultados de la innovación (2014)”. Infodatos. https://observatoriosociallacaixa.org/es/-/innovation-output-indicator-2014-

OCDE (2003). Manual de Frascati 2002. Medición de las actividades científicas y tecnológicas: propuesta de norma práctica para encuestas de investigación y desarrollo experimental. Madrid: Fecyt. ISBN: 8468828882

OCDE (2015a). OECD science, technology and industry scoreboard 2015: Innovation for growth and society. Paris: OECD Publishing.

https://doi.org/10.1787/sti_scoreboard-2015-en

OCDE (2015b). "Taxation of SMEs in OECD and G20 Countries". OECD tax policy studies, n. 23. Paris: OECD Publishing. https://doi.org/10.1787/9789264243507-en

OCDE (2006). Manual de Oslo. Guía para la recogida e interpretación de datos sobre innovación. Madrid: Grupo Tragsa. ISBN: 8461127811

https://doi.org/10.1787/9789264065659-es

Osuna-Alarcón, María R.; Rey-Rocha, Jesús; López-Navarro, Irene (2018). “Cultura científica e innovadora en la empresa española, en relación con el contexto educativo: primeras evidencias a partir de la encuesta sobre cultura científica, percepción y actitudes ante la ciencia y la innovación en el sector empresarial español”. En: Fernández-Bajón, María-Teresa; Sánchez-Jiménez, Rodrigo; Villaseñor-Rodríguez, Isabel (eds.). Conocimientos sin fronteras: colaboración científica e institucional en documentación e información. Madrid: Facultad de Ciencias de la Documentación. Universidad Complutense de Madrid, pp. 67-84. ISBN: 9788409021840

https://eprints.ucm.es/48994

Rey-Rocha, Jesús; López-Navarro, Irene (2016). Cuestionario CCe: Cultura científica, percepción y actitudes ante la ciencia y la innovación en el sector empresarial español. Estudio financiado por el Ministerio de Economía y Competitividad, Plan Estatal de Investigación Científica y Técnica y de Innovación, Proyecto CSO2014-53293-R.

http://digital.csic.es/handle/10261/171841

Rey-Rocha, Jesús; López-Navarro, Irene; Castro-Balaguer, Rafael; Cuesta-Santianes, María-José; Fernández-de-Pinedo, Nadia; González-Bravo, María-Isabel; Muñoz-Pérez, Félix; Osuna-Alarcón, María R.; Ribas-Mateos, Purificación; Sáiz-González, José-Patricio (2018). Informe CCe 2016. Cultura científica, percepción y actitudes ante la ciencia y la innovación en el sector empresarial español. Estudio financiado por el Ministerio de Economía y Competitividad, Plan Estatal de Investigación Científica y Técnica y de Innovación, Proyecto CSO2014-53293-R.

http://digital.csic.es/handle/10261/177927 
Ricyt (2015). Manual de Antigua: indicadores de percepción pública de la ciencia y la tecnología. Buenos Aires: Red Iberoamericana de Indicadores de Ciencia y Tecnología. ISBN 9789872044336

https://www.oei.es/historico/cienciayuniversidad/?article6361

Rojo, Raquel; Gómez-Caridad, Isabel (2006). “Análisis de la producción científica y tecnológica de la industria española en el sector de las tecnologías de la información y de las comunicaciones". El profesional de la información, v. 15, n. 3, pp. 190-201.

http://www.elprofesionaldelainformacion.com/contenidos/2006/mayo/4.pdf

Uyarra, Elvira (2006). Monitoring and analysis of policies and public financing instruments conducive to higher levels of R\&D investments The "Policy mix" Project. Country review Spain. Innovation policy in Spain. United Nations University; Universiteit Maastricht.

Vértesy, Daniel (2017). The innovation output indicator 2017: Methodology report. Luxembourg: Publications Office of the European Union. ISBN: 9789279764745

https://doi.org/10.2760/971852

Wilson, Daniel (2003). “Where to find the productivity gains from innovation?". FRBSF: Economic letter, n. $2003-04$. https://www.frbsf.org/economic-research/files/el2003-04.pdf

World Economic Forum (2019). The global competitiveness report 2018.

https://www.weforum.org/reports/the-global-competitveness-report-2018

Wynne, Brian (1991). "Knowledges in contexts". Science, technology and human values, v. 16, n. 1, pp. 111-121.

https://doi.org/10.1177/016224399101600108

\section{Colección de libros de bolsillo El profesional de la información (Editorial UOC) Últimos títulos publicados}
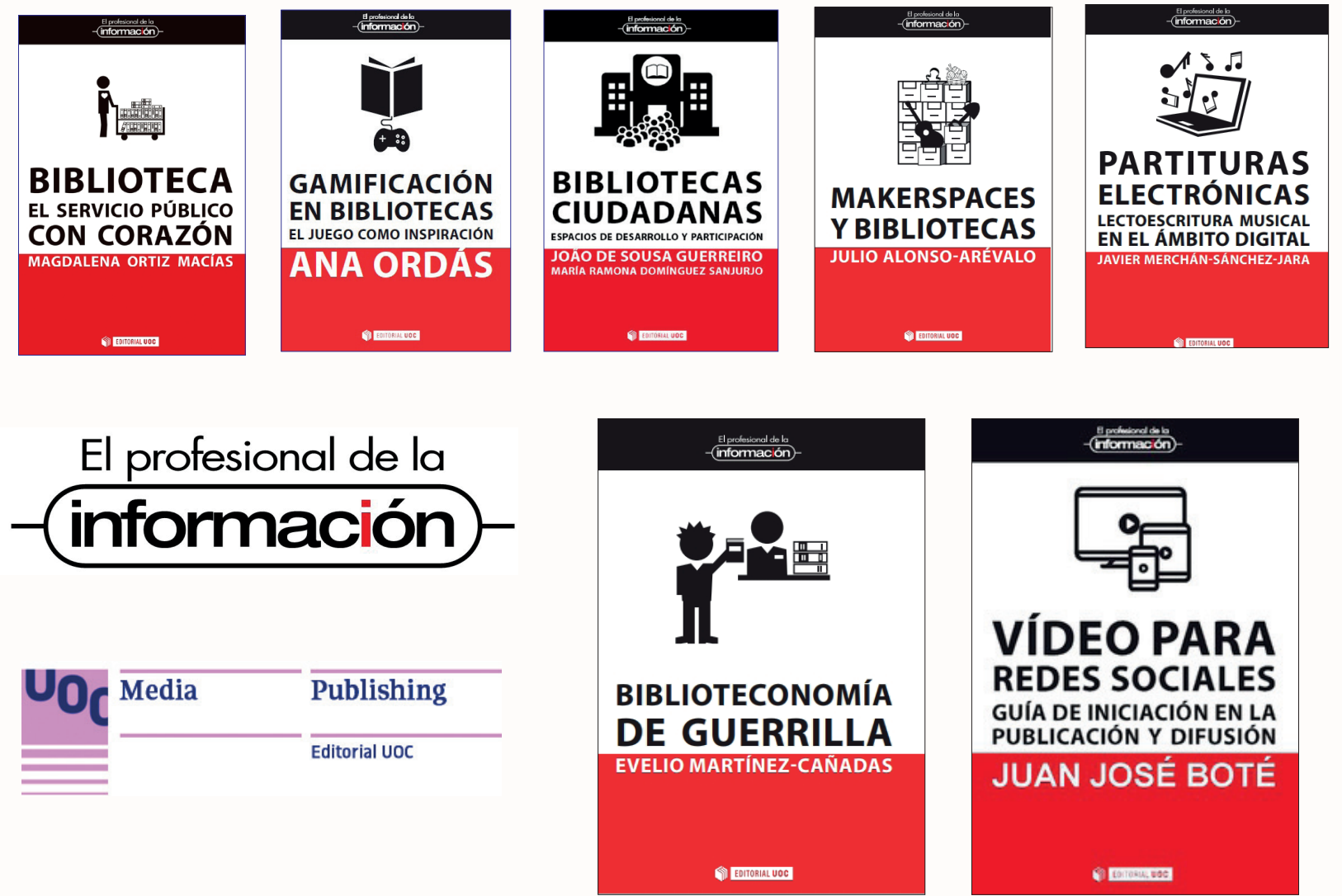

Más información:

http://www.elprofesionaldelainformacion.com/libros.html 